\title{
THRESHOLD GROWTH DYNAMICS
}

\author{
JANKO GRAVNER AND DAVID GRIFFEATH
}

\begin{abstract}
We study the asymptotic shape of the occupied region for monotone deterministic dynamics in $d$-dimensional Euclidean space parametrized by a threshold $\theta>0$, and a Borel set $\mathcal{N} \subset \mathbb{R}^{d}$ with positive and finite Lebesgue measure. If $A_{n}$ denotes the oocupied set of the dynamics at integer time $n$, then $A_{n+1}$ is obtained by adjoining any point $x$ for which the volume of overlap between $x+\mathscr{N}$ and $A_{n}$ exceeds $\theta$. Except in some degenerate cases, we prove that $n^{-1} A_{n}$ converges to a unique limiting "shape" $L$ starting from any bounded initial region $A_{0}$ that is suitably large. Moreover, $L$ is computed as the polar transform for $1 / w$, where $w$ is an explicit width function that depends on $\mathscr{N}$ and $\theta$.

It is further shown that $L$ describes the limiting shape of wave fronts for certain cellular automaton growth rules related to lattice models of excitable media, as the threshold and range of interaction increase suitably. In the case of box $\left(l^{\infty}\right)$ neighborhoods on $\mathbb{Z}^{2}$, these limiting shapes are calculated and the dependence of their anisotropy on $\theta$ is examined. Other specific two- and three-dimensional examples are also discussed in some detail.
\end{abstract}

\section{INTRODUCTION}

For motivation, we begin by considering a simple cellular automaton (CA) on the two-dimensional integer lattice $\mathbb{Z}^{2}$. Let $N=\left\{\left(x_{1}, x_{2}\right) \in \mathbb{Z}^{2}:\left|x_{1}\right| \leq 1\right.$ and $\left.\left|x_{2}\right| \leq 1\right\}$ be the so-called Moore neighborhood containing the origin 0 and the 8 lattice points closest to $\mathbf{0}$, and let $x+N$ be the translated neighborhood for $x \in \mathbb{Z}^{2}$. At each nonnegative integer time $n$, our automaton occupies a finite subset $A_{n}$ of the lattice. This occupied set changes over time according to the following dynamics:

(i) If $x \notin A_{n}$ and $\#\left(A_{n} \cap(x+N)\right) \geq 3$, then $x \in A_{n+1}$;

(ii) If $x \in A_{n}$, then $x \in A_{n+1}$.

In words, an unoccupied site becomes occupied next time if at least 3 of its 8 neighbors are occupied this time, while occupied sites are never vacated. This CA agrees with John Conway's celebrated Game of Life (cf. [7, 12]) except for two changes that greatly simplify the evolution. First, "birth" in (1.1) requires at least 3 living neighbors whereas Life needs exactly 3. Second, "death" is impossible in (1.1), but plays a key role in the complexity of Conway's dynamics.

Received by the editors October 19, 1991.

1991 Mathematics Subject Classification. Primary 52A99; Secondary 05B40, 60K35.

Key words and phrases. Growth model, threshold dynamics, excitable medium, polar transform. This research was partially supported by research grants from the National Science Foundation. 
Consequently our rule has two monotonicity properties, easily verified, that make it much more amenable to mathematical analysis. Namely, if we start from a finite set $A$ at time 0 and let $T$ denote the iterated transformation that gives rise to (1.1), then the following facts are self-evident:

$$
\begin{gathered}
A \subset T A ; \\
\text { If } A \subset B, \quad \text { then } T A \subset T B .
\end{gathered}
$$

That is, the occupied set can only grow, and if we start with more occupied sites initially then we have more occupied sites for all time.

So how does $A_{n}=T^{n} A$ evolve? Starting from a singleton (e.g., $\{\boldsymbol{0}\}$ ), or a set with very small connected components, no changes are possible since the threshold for birth is 3 . But suppose the initial configuration contains a sufficient local concentration of sites to promote birth at the boundary. For instance, choose $A=N$. Then $A_{n}$ spreads over $\mathbb{Z}^{2}$ at a constant rate, as indicated by Figure 1, quickly attaining an octagonal shape. (Each shaded "ring" depicts two successive time steps.) Since asymptotic shape is the central theme of this paper, let us now present a precise formulation of the limiting behavior we wish to establish. It is most convenient to embed the lattice in Euclidean space. Then for any Borel $A \subset \mathbb{R}^{d}$, and $\varepsilon>0$, write $A^{\varepsilon}=\left\{y \in \mathbb{R}^{d}:\|y-x\| \leq \varepsilon\right.$ for some $x \in A\}$. ( $\|\cdot\|$ is the Euclidean norm.) In this context, convergence of sets will be defined as follows.

Definition. A sequence of Borel sets $A_{n} \subset \mathbb{R}^{d}$ converges to a closed set $A \subset \mathbb{R}^{d}$ $\left(A_{n} \stackrel{H}{\rightarrow} A\right)$ iff given any $\varepsilon>0$, there is an $n_{\varepsilon}<\infty$ such that $A \subset A_{n}^{\varepsilon}$ and $A_{n} \subset A^{\varepsilon}$ for all $n \geq n_{\varepsilon}$.

This notion is usually called convergence in the Hausdorff metric. We require a closed limit set to ensure uniqueness.

Returning to our illustrative example, let $\mathscr{O}$ denote the octagonal region of $\mathbb{R}^{2}$ that is the closed convex hull of the 8 points: $\left( \pm \frac{1}{2}, 0\right),\left(0, \pm \frac{1}{2}\right),\left( \pm \frac{1}{3}, \pm \frac{1}{3}\right)$. Figure 1 indicates that as $n \rightarrow \infty$,

$$
n^{-1} T^{n} N \stackrel{H}{\rightarrow} \mathscr{O} \text {. }
$$

In words, the shape $A_{n}$ spreads linearly with asymptotic shape $\mathscr{O}$. For this particular example the claimed convergence is easy to check because the exact form of the $n$th iterate is readily obtained by induction. Moreover, starting from any finite set $A$ that contains a translate of $N$,

$$
n^{-1} T^{n} A \stackrel{H}{\rightarrow} \mathscr{O},
$$

so the limit is independent of the initial configuration provided it is sufficiently large. Claim (1.5) follows quite easily from (1.2)-(1.4); for now, this argument is left as a little puzzle.

Note that the birth threshold in rule (1.1) equals $\frac{1}{3}$ the size of the neighbor set. Suppose next that we change to a 10-box neighbor set $N=\left\{\left(x_{1}, x_{2}\right) \in\right.$ $\mathbb{Z}^{2}:\left|x_{1}\right| \leq 10$ and $\left.\left|x_{2}\right| \leq 10\right\}$, while increasing the threshold from 3 to $\theta=$ $\frac{1}{3} \# \mathscr{N}=\frac{1}{3}(21)^{2}=147$. Figure 2 shows the resulting pattern of growth starting from the new $N$. (Individual cells are smaller in the second figure than in the first.) Evidently the range-10 example also attains an asymptotic shape. The exact behavior of this CA is much more complicated than (1.1), however. 


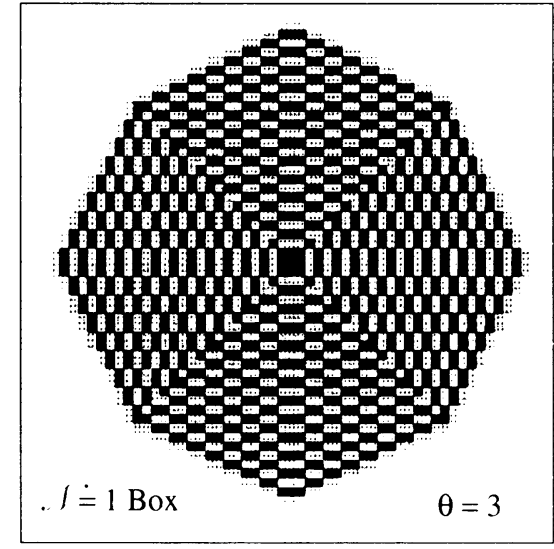

FIGURE 1

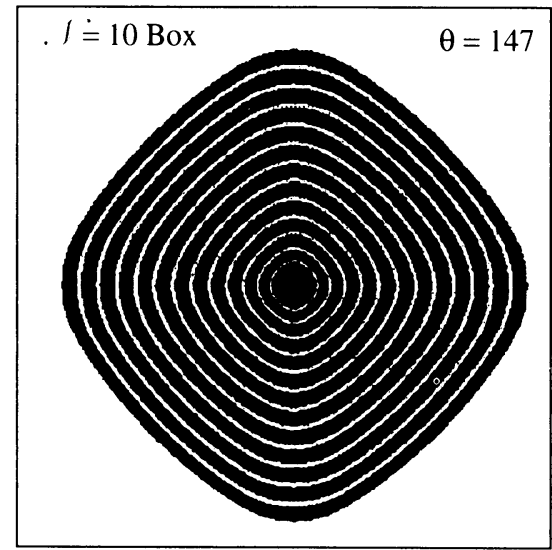

FIGURE 2

Although we will prove later that it does have an asymptotic shape, i.e., that $n^{-1} T^{n} A$ converges to a unique limit from all suitably large finite initial $A$, we do not know a straightforward way to characterize the limit, nor even whether it is a polygon.

The real message of Figure 2 is not the quagmire of large-scale lattice rules, but rather its "smoother" shape, suggesting a continuum limit as the threshold $\theta$ and range $\rho$ of the $\rho$-box neighbor set increase appropriately. More specifically, for positive integer values of $\theta$ and $\rho$ write

$$
N_{\rho}=\left\{\left(x_{1}, x_{2}\right) \in \mathbb{Z}^{2}:\left|x_{1}\right| \leq \rho \text { and }\left|x_{2}\right| \leq \rho\right\}
$$

and define $T=T_{\theta, \rho}$ on finite subsets of $\mathbb{Z}^{2}$ by

$$
T A=\left\{x \in \mathbb{Z}^{2}: \#\left(A \cap\left(x+N_{\rho}\right)\right) \geq \theta\right\} \cup A .
$$

Assume that a sequence of thresholds $\theta_{k}$ and a sequence of ranges $\rho_{k}$ both tend to $\infty$ in such a way that $\theta_{k} / \rho_{k}^{2} \rightarrow \theta$. Then Riemann approximation suggests that transformations $\rho_{k}^{-1} T_{\theta_{k}, \rho_{k}}$ approach a limiting transformation $\mathscr{T}=\mathscr{T}_{\theta}$ on nice sets, with

$$
\mathscr{T} A=\{x:|A \cap(x+\mathcal{N})| \geq \theta\} \cup A .
$$

Here $\mathscr{N}=\left\{\left(x_{1}, x_{2}\right) \in \mathbb{R}^{2}:\left|x_{1}\right| \leq 1\right.$ and $\left.\left|x_{2}\right| \leq 1\right\}$ is the Euclidean box of side 2 centered at $\mathbf{0}$, and $|\cdot|$ denotes Lebesgue measure when applied to sets. The precise meaning of this convergence of transformations will be spelled out toward the end of the paper. But the idea is simple enough that this informal sketch should serve to motivate our study of Euclidean threshold growth dynamics: iteration of rule (1.7) or its extension to $d$-dimensional space and any Borel neighbor set $\mathscr{N}$ with $0<|\mathcal{N}|<\infty$.

Our central problem, then, is as follows. Given $\theta>0$ and a bounded Borel set $\mathscr{N} \subset \mathbb{R}^{d}$ of positive measure, with $\mathscr{T}$ defined by (1.7), is there a limit set $L \subset \mathbb{R}^{d}$ such that

$$
n^{-1} \mathscr{T}^{n} A \stackrel{H}{\rightarrow} L
$$

as $n \rightarrow \infty$, for all sufficiently large bounded initial sets $A \subset \mathbb{R}^{d}$, and is it possible to characterize $L$ ? As we shall see, this problem can be solved in great 
generality. Moreover, in specific examples such as the threshold-range limit corresponding to Figures 1 and 2 (i.e., $d=2, \mathcal{N}=$ box of side $2, \theta=\frac{4}{3}$ ), the asymptotic shape can be calculated quite explicitly. Most of the remainder of this introduction will be devoted to a discussion of our main result and a broad outline of its proof. But first, let us briefly digress to mention the initial motivation for this work: cellular automaton modeling of excitable media.

Mathematical models for excitable media attempt to capture and explain the key features of periodic wave transmission through environments such as a network or tissue. Since the pioneering work of N. Wiener and A. Rosenbluth [13] in the mid-1940s, a great many researchers across the spectrum of applied science have adopted various modeling frameworks, most notably partial differential equations, cellular automata, and coupled lattice maps. A common feature of many such models is the requirement that some threshold level of excitation occur in a neighborhood of a location in order for that location to become "excited" and conduct a pulse. Such activity is typically followed by a refractory period in which further excitation is inhibited. Physical systems that exhibit this basic phenomenology include neural networks, cardiac muscle, and the Belousov-Zhabotinsky (BZ) oscillating chemical reaction. In two dimensions such systems are typically characterized by the emergence of spatially-distributed stable "target" patterns or spirals.

Arguably the simplest dynamical system that emulates an excitable medium is a 3-stage, range-1, threshold-1 CA known as the Greenberg-Hastings (GH) model (cf. [8]). With the advent of effective computer visualization technology there has been a recent flurry of excitable CA modeling, based on the GH prototype but trying to approximate precise quantitative features of observed phenomena (e.g., curvature and wave velocity in the $\mathrm{BZ}$ reaction). To accomplish this, most experimentalists introduce several rather ad hoc parameters that are designed to generate an assortment of nonlinear effects.

Together with $\mathrm{R}$. Fisch, we recently carried out a detailed experimental study of a 3-parameter family of simple GH-type rules [6]. The parameters are $\rho$ : the range of interaction (assuming a box neighborhood); $\theta:$ the threshold number of excited neighbors required for a cell to become excited; and $\kappa$ : the number of possible states (=colors) per cell. Here state 0 is "rested," 1 is "excited," and $2 \ldots \kappa-1$ are refractory. The refractory states update automatically each time, so $\kappa$ governs the recovery time. A more detailed description of these $\mathrm{GH}$ dynamics will be presented in the last section of this paper.

Our main findings in [6] were two-fold. First we discovered that as one varies $\rho, \theta$ and $\kappa$, this family of models displays a remarkably complex phase portrait containing several cutoffs that divide the ergodic behavior of the infinite lattice dynamics into qualitatively distinct regimes. For instance, one regime is characterized by statistical noise, another by nucleation of stable spiral pairs, a third by clustering of aligned parades of wave fragments, and a fourth by global relaxation. See [6] for color computer graphics that illustrate these various forms of complex self-organization, and for an extensive bibliography of research on excitable media. The even more exotic dynamics of closely-related cyclic cellular automaton (CCA) models are also described and illustrated. IBM-compatible freeware [5] that facilitates $\mathrm{GH}$ and CCA experimentation is available from the authors. 
Our second discovery was the emergence of curvature and limiting dynamics in excitable CA systems, as the threshold and range increase, with $\theta_{k} / \rho_{k}^{2} \rightarrow \theta$. This threshold-range scaling is particularly appealing from a mathematical point of view since the limiting Euclidean evolutions are surprisingly amenable to rigorous analysis. For instance, one can investigate the geometry of wave fronts and spiral curves in the threshold-range limit. When $\theta$ is small, as it turns out, the asymptotic shapes of propagating Euclidean $\mathrm{GH}$ rings and spirals are precisely the same as the asymptotic shape (1.8) for threshold growth dynamics. This application was the original motivation for our present study; we will discuss it further at the conclusion of the paper.

Let us now turn to the strategy for proving a limiting shape theorem of the form (1.8). It is instructive to consider first the one-dimensional situation. The shape result is little more than an exercise when $d=1$, but provides a useful template for the challenge of higher dimensions. Suppose that $\mathscr{N}$ is symmetric about 0 , with $\sup \mathscr{N}=l<\infty$. If $\theta \geq \frac{1}{2}|\mathscr{N}|$, then starting from any bounded $A$ growth is impossible beyond the extreme points of $A$, and so (1.8) holds with $L=\{0\}$ (i.e., the limiting shape is trivial). On the other hand, suppose $\theta<\frac{1}{2}|\mathcal{N}|$, and start from $A=[-l, l]$. Then with each iteration the occupied interval grows at both edges by a constant amount $w>0$, where

$$
w=\max \{x \geq 0:|(x+\mathscr{N}) \cap(-\infty, 0]| \geq \theta\} .
$$

Hence $L=[-w, w]$ in this case. As in our first CA example, (1.3) implies the same limiting shape from any bounded $A$ that contains a translate of $[-l, l]$. For general $\mathscr{N}$ in one dimension one needs to identify two edge velocities:

$$
\begin{aligned}
& w^{+}=\max \{x \geq 0:|(x+\mathcal{N}) \cap(-\infty, 0]| \geq \theta\} \\
& w^{-}=\max \{x \geq 0:|(-x+\mathcal{N}) \cap[0, \infty)| \geq \theta\} .
\end{aligned}
$$

If either set of $x$ values is empty the corresponding max is defined to be 0 . Whenever $A$ contains a translate of $[-l, l]$, it is straightforward to check that (1.8) holds with $L=\left[-w^{-}, w^{+}\right]$. Further details are left to the reader. For the remainder of the paper we assume that $d \geq 2$.

Since $L$ is determined by half-line velocities (1.9) in one dimension, it is natural to describe the limiting shape in higher dimensions by means of the asymptotic velocity $\alpha(u)=\alpha_{L}(u)$ for each unit vector $u$ in the $d-1$ dimen-

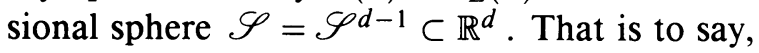

$$
L=\{\lambda u: u \in \mathscr{S}, 0 \leq \lambda \leq \alpha(u)\} .
$$

This approach is well-established in the theory of stochastic growth models. Explicit computation of the limit shape is possible for a few processes, e.g., branching random walks in which case $L$ is described by means of a Legendre transform [1], or internal diffusion limited aggregation [9] for which one can apply probabilistic harmonic analysis to prove that $L$ is a Euclidean ball. More often, the subadditive ergodic theorem is used to prove existence of radial limits for the rate of growth. Various techniques are then employed to "patch together" the $\alpha(u)$ into a convex limiting shape. This strategy applies to Eden's model [3] and other prototypes for random contagion; Durrett [2] gives a very nice introduction to the subject. It should be noted, however, that subadditivity rarely reveals quantitative information about the limiting shape, e.g., whether or not it is isotropic (i.e., a Euclidean ball). In $\S 6$ we will mimic the 
stochastic approach to prove an implicit shape theorem for threshold growth on $\mathbb{Z}^{d}$. But for Euclidean threshold growth one can obtain a much more concrete representation.

The idea behind our identification of $L$ in the Euclidean setting is quite simple, and familiar to researchers in the field of interface dynamics. Any growing cluster with an asymptotic shape becomes locally flat at its boundary. Since our threshold growth rule is effectively local, unoccupied sites near the boundary of $\mathscr{T}^{n} A$ eventually encounter a configuration that is virtually indistinguishable from a half-space. Given $u \in \mathscr{S}$, denote the half-space

$$
H_{u}^{-}=\left\{x \in \mathbb{R}^{d}:\langle x, u\rangle \leq 0\right\} .
$$

$(\langle\cdot, \cdot\rangle$ is the Euclidean dot product.) In clear analogy with the one-dimensional case, $H_{u}^{-}$propagates at constant speed

$$
w(u)=\max \left\{\lambda \geq 0:\left|(\lambda u+\mathscr{N}) \cap H_{u}^{-}\right| \geq \theta\right\}
$$

Not surprisingly this width function $w$ plays a key role in our evaluation of the limiting shape. But how do $\alpha$ and $w$ "patch together"? Assume for simplicity that $\alpha$ is continuously differentiable $\left(\mathscr{C}^{1}\right)$. The half-space encountered at the boundary in direction $u$ is $H_{\nu(u)}^{-}$, where $\nu(u)$ denotes the (outward) normal to $L$ at the point $\alpha(u) u$. This half-space has speed $w(\nu(u))$ in direction $\nu(u)$, which yields speed $w(\nu(u)) /\langle u, \nu(u)\rangle$ in direction $u$. We conclude that

$$
\alpha(u)=\frac{w(\nu(u))}{\langle u, \nu(u)\rangle}
$$

Since $\nu(u)$ depends on $L,(1.12)$ determines the limiting shape implicitly. When $d=2$ this formula is equivalent to a nasty differential equation for $\alpha$ (in polar coordinates, say). For many simple choices of $\mathscr{N}$ the solution exhibits singularities and bifurcations indicating that $L$ may not be $\mathscr{C}^{1}$. In that case, how convincing is our heuristic? The solution to this quandary involves a representation of $\alpha$, agreeing with (1.12) in the $\mathscr{C}^{1}$ case, in terms of the polar transform. For any $K \subset \mathbb{R}^{d}$, the polar of $K$ is the set

$$
K^{*}=\left\{x \in \mathbb{R}^{d}:\langle x, y\rangle \leq 1 \text { for all } y \in K\right\} .
$$

This is the mapping that takes the unit ball in a Banach space to the unit ball of the dual space. Also, for any continuous $f: \mathscr{S} \rightarrow[0, \infty]$, we denote the "shape corresponding to $f$ " as

$$
K_{f}=\{\lambda u: u \in \mathscr{S}, 0 \leq \lambda \leq f(u)\}
$$

Recall that $L=K_{\alpha}$ by definition. For smooth $\alpha,(1.12)$ is equivalent to

$$
L=K_{1 / w}^{*}
$$

as explained in the Appendix to this paper (cf. (A.3)-(A.5)).

Our main goal is to demonstrate that Euclidean threshold growth dynamics attain a limiting shape $L$ in great generality: if the polar of $K_{1 / w}$ is either $\{0\}$ (trivial) or has full dimension $d$, then (1.8) holds with $L$ given by formula (1.15). More precisely, we will prove the following result. 
Theorem 1. Let $\mathscr{N} \in \mathscr{B}\left(\mathbb{R}^{d}\right)$ be a set (bounded or unbounded) with finite volume, and fix $0<\theta<\infty$. Let $\mathscr{T}^{n} A$ denote the nth iterate of Euclidean threshold growth dynamics with neighbor set $\mathcal{N}$ and threshold $\theta$, started from A. With $w$ defined by (1.11), assume there is a direction $u \in \mathscr{S}^{d-1}$ such that

$$
\left.w\right|_{H_{u}^{-} \cap \mathscr{S}^{d-1}}>0 .
$$

There exists an $m<\infty$ so that if $A \in \mathscr{B}\left(\mathbb{R}^{d}\right)$ is bounded and $B(m) \subset A$, then the dynamics grow linearly with a d-dimensional limiting shape given by the polar transform (1.13)-(1.14) for $1 / w$ :

$$
n^{-1} \mathscr{T}^{n} A \stackrel{H}{\rightarrow} K_{1 / w}^{*}
$$

as $n \rightarrow \infty$. Limit law (1.17) also holds if the polar for $1 / w$ is completely degenerate, i.e., $K_{1 / w}^{*}=\{\mathbf{0}\}$.

In the Appendix, we show that the half-space condition (1.16) is equivalent to full dimension $d$ (i.e., nonempty interior) for $K_{1 / w}^{*}$. Thus only in partially degenerate cases, when the dimension of the polar is less than $d$ but greater than 0 , does the shape theorem break down. In some such cases it must be reinterpreted, while others provide genuine counterexamples. Such counterexamples, and the fact that in two dimensions the boundary of $L$ is $\mathscr{C}^{1}$ only if $K_{1 / w}$ is strictly convex, indicate a need for care while fashioning the halfspace heuristic into an honest theorem.

To conclude this introduction, let us summarize the organization of the remainder of the paper. For further motivation, $\S 2$ presents several concrete examples of Theorem 1 . We begin by examining in considerable detail the twodimensional case where $\mathscr{N}$ is a box of width 2 centered at the origin since, as explained earlier, the resulting limit shapes are related to the behavior of threshold growth cellular automata with large range. The width functions $w_{\theta}$ are computed explicitly and the anisotropy of $L_{\theta}$ is examined as $\theta$ varies. None of these shapes is $\mathscr{C}^{1}$, but for certain values of $\theta$ the deviation from a circle is remarkably small. These box neighborhood shapes illustrate certain regularity properties of $L$ that are peculiar to two dimensions. For example, it turns out that $L$ is always "smooth" (i.e., $\alpha$ is $\mathscr{C}^{1}$ ) if $\mathscr{N}$ is strictly convex, $\mathscr{N}=-\mathscr{N}$, and $d=2$; we conjecture that this is not always the case for $d \geq 3$. Also in $d=2$, if $\mathscr{N}$ is the unit ball of $l^{p}$ for $p \in[1, \infty]$, then the limit of shapes $L_{\theta}$ as $\theta \rightarrow \frac{1}{2}|\mathscr{N}|$ is the unit ball of the dual space $l^{q}\left(\frac{1}{p}+\frac{1}{q}=1\right)$. This result definitely does not extend to higher dimensions, but was the clue that first led us to understand the role of the polar. As another interesting twodimensional example, we take $\mathcal{N}$ to be an equilateral triangle region centered at $\mathbf{0}$, in which case $K_{1 / w}$ is not convex. The graphs of $K_{1 / w}$ and $K_{1 / w}^{*}$ illustrate this counter-intuitive situation. Our final examples deal with degenerate cases when the polar of $1 / w$ is nontrivial but the hypothesis of Theorem 1 is not satisfied. Simple two-dimensional neighbor sets show that (i) the limiting shape can depend on the initial configuration, or (ii) the occupied set can grow at a sublinear rate. Also, a prism neighborhood in $\mathbb{R}^{3}$ shows that growth can remain confined to a bounded region even though $K_{1 / w}^{*}$ is nontrivial, providing a bona fide counterexample to (1.15). 
In $\S 3$ we use the width function $w$ and monotonicity to show the easy half of the main result in complete generality:

$$
n^{-1} \mathscr{T}^{n} A \subset\left(K_{1 / w}^{*}\right)^{\varepsilon}
$$

for any bounded $A \subset \mathbb{R}^{d}$ and all sufficiently large $n$. One consequence of this upper bound is a trivial limit $(L=\{\boldsymbol{0}\})$ whenever $\theta$ exceeds $\frac{1}{2}|\mathcal{N}|$. The proof of Theorem 1 is completed in $\S 4$. Namely, we show that condition (1.16) implies the opposite inclusion

$$
\left(n^{-1} \mathscr{T}^{n} A\right)^{\varepsilon} \supset K_{1 / w}^{*}
$$

for all sufficiently large $n$, establishing (1.17) in all but partially degenerate cases. For the sake of clarity, some preliminaries, several properties of the threshold growth transformations $\mathscr{T}=\mathscr{T}_{\theta, \mathcal{N}}$, and a number of standard facts about polar transforms have been relegated to the Appendix. Readers are encouraged to confront these technical details as the need arises and energy permits.

In the final two sections of the paper we return to cellular automata such as (1.1). A limiting shape result is proved for such CA growth rules with arbitrary threshold and range, assuming certain symmetries of the neighbor set $N$. Since subadditivity is our main tool in the lattice setting we obtain no detailed information about $L$; it is simply the unit ball with respect to some implicitly defined norm. Nevertheless, by appealing to the threshold-range scaling that connects lattice transformations $T$ such as (1.6) with a limiting Euclidean transformation $\mathscr{T}$ such as (1.7), we prove that the corresponding implicit asymptotic lattice shapes converge to a shape described by our formula (1.15). For instance, the shape $L_{4 / 3}$ for the Euclidean box neighborhood that is detailed in the next section provides a very good approximation to the outermost ring generated by the range-10 CA in Figure 2. Both the CA shape result and the threshold-range scaling result are presented in $\S 5$ (Theorems 2 and 3, respectively). The paper concludes, in $\S 6$, with a brief discussion of excitable cellular automata and their connections to threshold growth dynamics. We define the Greenberg-Hastings and Cyclic CA models that were mentioned earlier and explain why the rings they generate have the same geometry as the shapes generated by our transformations $T$ provided the threshold $\theta$ is sufficiently small. However excitable ring dynamics are not monotone, so new and challenging problems arise.

\section{EXAMPLES IN TWO AND THREE DIMENSIONS}

We will now discuss the asymptotic shape of threshold growth dynamics in six specific settings where $d=2$ or $d=3$. Our examples are designed to illustrate (i) explicit quantitative content of the polar formula in concrete cases when $K_{1 / w}^{*}$ is nondegenerate, and (ii) subtleties and counterexamples to the polar formula in some degenerate cases. Example 2 describes two easy corollaries of Theorem 1 that are illustrated by Example 1.

Example 1. Consider the two-dimensional box neighborhood that figured in our introduction: $\mathscr{N}=\left\{x \in \mathbb{R}^{2}:\|x\|_{\infty} \leq 1\right\}$. Let us compute the width functions $w_{\theta}$, and graph the limiting shapes $L_{\theta}$, as $\theta$ increases from 0 to $\theta_{c}=2$. We parametrize $\mathscr{S}^{1}$ in terms of the polar coordinate $\varphi \in[0,2 \pi)$. By symmetry, it suffices to compute $w_{\theta}(\varphi)$ for $0 \leq \varphi \leq \pi / 4$. Our undergraduate research 
assistant David Perry carried out this rather arduous trigonometric calculation. The result, for $0<\theta<2$, is

$$
w_{\theta}(\varphi)= \begin{cases}\left(1-\frac{\theta}{2}\right) \cos \varphi & \text { if } \varphi \leq \arctan \frac{\theta}{2} \\ \cos \varphi+\sin \varphi-\sqrt{\theta \sin 2 \varphi} & \text { if } \arctan \frac{\theta}{2} \leq \varphi \leq \frac{\pi}{4}\end{cases}
$$

Note that $K_{1 / w_{\theta}}$ has $\mathscr{C}^{1}$ boundary, but is not strictly convex (e.g., near $\varphi=$ 0 ). Thus, by Proposition A2(f) of the Appendix, none of the limiting shapes $L_{\theta}$ for the square has a differentiable boundary. As noted in the final paragraph of the Appendix, this is caused by the flat edges of $\mathscr{N}$. In contrast, all the limiting shapes for the neighborhoods $\mathscr{N}_{p}=\left\{x \in \mathbb{R}^{2}:\|x\|_{p} \leq 1\right\}, 1<p<\infty$, are $\mathscr{C}^{1}$. That same discussion also implies that all shapes $L_{\theta, p}, 1 \leq p \leq \infty$, are strictly convex.

If $K$ is a two-dimensional bounded convex set, with $\mathscr{C}^{1}$ boundary represented by $r=r(\varphi)$, and $r^{*}(\varphi)=\alpha_{K^{*}}(\varphi)$, then

$$
r^{*}\left(\varphi-\arctan \frac{r^{\prime}(\varphi)}{r(\varphi)}\right)=\sqrt{\left[\left(\frac{r^{\prime}(\varphi)}{r(\varphi)}\right)^{2}+1\right] \cdot \frac{1}{r(\varphi)^{2}}}
$$

Put $r=1 / w$ to get

$$
\alpha_{L}\left(\varphi+\arctan \frac{w^{\prime}(\varphi)}{w(\varphi)}\right)=\sqrt{w(\varphi)^{2}+w^{\prime}(\varphi)^{2}}
$$

Plugging in (2.1), we conclude that $\alpha_{L}$ is given by

$$
\alpha_{L}(\psi(h))=\sqrt{2+\frac{\theta}{\sin 2 h}-2 \sqrt{\theta} \cdot \frac{\sin h+\cos h}{\sqrt{\sin 2 h}}},
$$

for $\arctan \frac{\theta}{2} \leq h \leq \frac{\pi}{4}$, where

$$
\psi(h)=h-\arctan \left(\frac{\tan h-1+\sqrt{\theta} \cdot\left[\left(1-\tan ^{2} h\right) / \sqrt{2 \tan h}\right]}{\tan h+1-\sqrt{2 \theta \tan h}}\right) .
$$

Note that $\alpha_{L_{\theta}}(0)=1-\frac{\theta}{2}$, and $\alpha_{L_{\theta}}\left(\frac{\pi}{4}\right)=\sqrt{2}-\sqrt{\theta}$.

In Figure 3 we have plotted $L_{\theta}$ for $\theta$ increasing from .1 to 1.9 in increments of .1. Of course the sizes decrease as $\theta$ increases. In so doing the shapes interplate between the square $\mathcal{N}$ and a "diamond." Notice that for $\theta \approx .3$ the shape is almost circular. In fact, if $B=B_{\theta}$ denotes the best-fit ball, then at $\theta=.35$ we find that $\sup \left|\alpha_{L}(\varphi)-\alpha_{B}\right| / \alpha_{B}<.011$. None of these shapes is precisely a ball since the boundary of each $L_{\theta}$ has discontinuous derivative at $\varphi=0$. But the fit is sometimes close enough that corresponding lattice cellular automata might appear to generate circular wave fronts. For instance, Greenberg-Hastings rules with fairly small range $\rho$ and threshold $\theta$, where $\theta / \rho^{2} \approx .35$ give this (false!) impression. Evidently the issue of isotropic limiting shape is very delicate for lattice growth dynamics. Internal diffusion limited aggregation [9] is one (random) lattice rule that spreads like a Euclidean ball. But despite the claims of some experimentalists, we suspect that in CA models for excitable media the limiting shape of a wave front invariably exhibits residual anisotropy from the underlying lattice. 


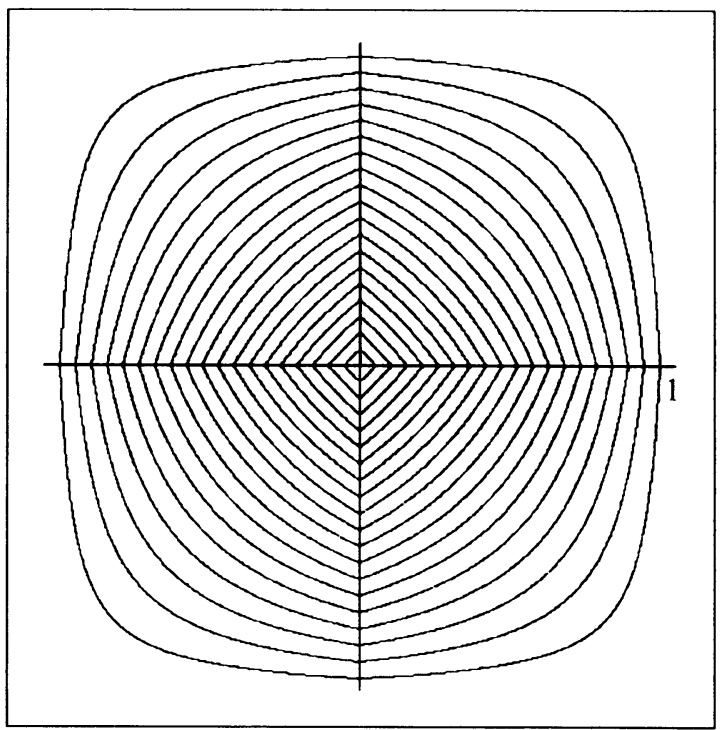

FIGURE 3

We conclude our discussion of the two-dimensional square neighborhood with a problem for the reader: describe $\lim _{\theta \rightarrow 0} L_{\theta, p}$ for the neighborhoods $\mathscr{N}_{\theta, p}, 0<p \leq 1$.

Example 2. Let $\mathscr{N}$ be bounded and convex. Assume that $\alpha_{\mathscr{N}}>0$ on $H_{u}^{-}$for some $u \in \mathscr{S}$. Then (1.16) holds for all sufficiently small $\theta>0$; let $L_{\theta}$ be the corresponding limit shapes. What is $L_{0}=\lim _{\theta \rightarrow 0} L_{\theta}$ ? If $\theta \approx 0+$, then

$$
w(u) \approx S_{\mathscr{N}}(-u)=S_{-\mathscr{N}}(u) ;
$$

hence $1 / w \approx \alpha_{-N^{*}}$, and so

$$
L_{\theta}=K_{1 / w}^{*} \approx-\mathscr{N}^{* *}=-\mathscr{N} .
$$

(The $\approx$ can be justified using Proposition A2 $(g)$.$) In fact, it is not hard to$ see that $L_{0}=-\mathscr{N}$ is the limit shape for " $\theta=0$ dynamics" $\mathscr{T}_{0}$ given by

$$
\mathscr{T}_{0} A=\{x: A \cap(x+\mathscr{N}) \neq \varnothing\} \cup A .
$$

This result holds in any dimension. If $\mathscr{N}$ is not convex, but there is a $u \in \mathscr{S}$ such that $\left|H_{v}^{-} \cap \mathscr{N}\right|>0$ for all $v \in H_{v}^{-}$, then $L_{0}=-\operatorname{co}(\mathscr{N}) . L_{0}$ is still the limit shape for $\mathscr{T}_{0}$ as long as $\mathscr{N}$ contains no sets of measure 0 (e.g., isolated points) that can be separated from the rest of $\mathscr{N}$ by a half-plane. (Such sets play no role in $\mathscr{T}_{\theta}$ for $\theta>0$, but may clearly influence $\mathscr{T}_{0}$.)

Under the assumption that $\mathscr{N}$ is symmetric (i.e., $-\mathscr{N}=\mathscr{N}$ ), we will see in $\S \S 3$ and 4 that $L_{\theta}$ is nontrivial for $\theta<\theta_{c}=\frac{1}{2}|\mathscr{N}|$, and that $\lim _{\theta \uparrow \theta_{c}} L_{\theta}=\{0\}$. But what is the limiting shape of the set as it shrinks? In the case of Example 1, Figure 3 clearly suggests a diamond. Assume also that $d=2$ and $\mathcal{N}$ is also $\frac{\pi}{2}$ rotation symmetric. Then letting $u^{\perp}$ denote the (counter-clockwise) $\frac{\pi}{2}$-rotation of $u$, we get

$$
\theta_{c}-\theta \approx 2 \alpha_{\mathscr{N}}(u) w\left(u^{\perp}\right)=2 \alpha_{\mathscr{N}}(u) w(u) .
$$




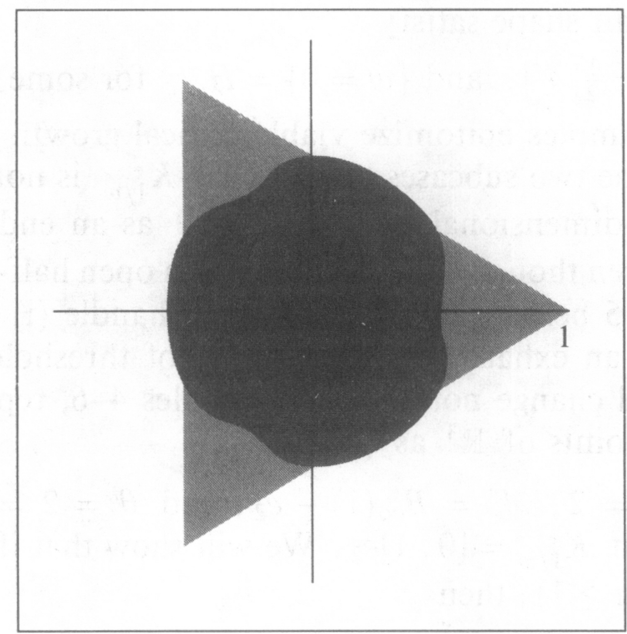

FIGURE 4

Hence

$$
\frac{1}{w(u)} \approx \frac{2}{\theta_{c}-\theta} \alpha_{\mathscr{N}}(u),
$$

and so

$$
\lim _{\theta \uparrow \theta_{c}} \frac{2}{\theta_{c}-\theta} L_{\theta}=\mathscr{N}^{*} .
$$

For instance the limiting shape for the $l^{p}$-ball $(1 \leq p \leq \infty)$, as $\theta \uparrow \theta_{c}$, is the $l^{q}$-ball $\left(\frac{1}{p}+\frac{1}{q}=1\right)$. Evidently, in the general symmetric case (2.2) holds with $\mathscr{N}$ replaced by its $\frac{\pi}{2}$-rotation. We have checked that this result does not extend to $d \geq 3$.

Example 3. Our neighbor set is the two-dimensional triangular region $\mathscr{N}=$ $\operatorname{co}\left(\left\{z \in \mathbb{C}: z^{3}=1\right\}\right)$. Let $\theta=.3$, say. In this case $1 / w$ is not convex, so $K_{1 / w}$ cannot be approximated by strictly convex sets. But Theorem 1 applies. Figure 4 shows $\mathscr{N}$ (light gray) and a numerical calculation of $K_{1 / w}$ (dark gray, not shown on the same scale) and $L=K_{1 / w}^{*}$ (black).

In two dimensions, the only situation not covered by Theorem 1 is the critical case $\theta=\frac{1}{2}|\mathcal{N}|$. (Our argument for this assertion anticipates results from the next section and the Appendix, so the reader may want to defer study of the remainder of this paragraph.) Proposition 1 in $\S 3$ will show that for $\theta>\frac{1}{2}|\mathcal{N}|$, $\mathscr{T}$ is convex-confined, meaning that to each bounded set $A \in \mathscr{B}\left(\mathbb{R}^{d}\right)$ there corresponds an $R<\infty$ such that $\mathscr{T}^{n} A \subset B(R)$ for all $n \geq 0$. If $\mathscr{T}$ is not convex-confined, then (A.6) implies that $\{w=0\} \subset H_{u}^{-}$for some $u$. Hence $\{w>0\} \supset \operatorname{in}\left(H_{-u}^{-}\right) \cap \mathscr{S}$. Choose $u^{\perp}$ orthogonal to $u$. As in the proof of Proposition 1, if $\theta<\frac{1}{2}|\mathcal{N}|$ then $w>0$ either in a neighborhood of $u^{\perp}$ or a neighborhood of $-u^{\perp}$. When $d=2$ (but not for $d>2$ !) this implies that $\{w>0\} \supset H_{-u \pm \delta}^{-} \cap \mathscr{S}$ for a suitable choice of sign and small $\delta>0$. But then hypothesis $(1.16)$ is satisfied. In the critical case, $w(v)>0$ forces $w(-v)=0$. So in fact, the only cases in $\mathbb{R}^{2}$ that are neither convex-confined nor have a 
two-dimensional limit shape satisfy

$$
\theta=\frac{1}{2}|\mathcal{N}|, \text { and }\{w=0\}=H_{u}^{-} \text {for some } u .
$$

Our next two examples epitomize viable critical growth in the plane. They are prototypes for the two subcases of (2.3): (i) $K_{1 / w}^{*}$ is not completely trivial, and hence is a one-dimensional interval with $\mathbf{0}$ as an endpoint; (ii) $K_{1 / w}^{*}$ is completely trivial even though $w$ is positive on an open half-space. The analysis of Examples 4 and 5 below can be extended to handle (i) and (ii) in general, thereby completing an exhaustive classification of threshold growth dynamics for $d=2$. We will change notation in Examples 4-6, representing points of $\mathbb{R}^{2}$ as $(x, y)$ and points of $\mathbb{R}^{3}$ as $(x, y, z)$.

Example 4. Let $d=2, \mathscr{N}=B_{\infty}(1)-e_{2}$, and $\theta=2=\frac{1}{2}|\mathcal{N}|$. Then it is routine to check that $K_{1 / w}^{*}=[0,1] e_{2}$. We will show that if $A_{m}=\{-m \leq x \leq$ $m,-1 \leq y \leq 1\} \quad(m \geq 1)$, then

$$
n^{-1} \mathscr{T}^{n} A \stackrel{H}{\rightarrow}\left[0, l_{m}\right] e_{2}
$$

for some $l_{m}<1$. This is our first counterexample to the polar formula for $L$. Nevertheless, $l_{m} \rightarrow 1$ as $m \rightarrow \infty$. In other words, a limit set arbitrary close to $K_{1 / w}^{*}$ can be achieved by starting from a sufficiently large finite set. The arguments for our convergence claims are as follows.

First, to show (2.4), consider the dynamics starting from $A_{m}^{\prime}=\{-m \leq x \leq$ $m, y \leq 1\}$. The given $\mathscr{N}$ satisfies (2.3) with $u=e_{2}$, so growth is only possible in directions $v$ with $\left\langle v, e_{2}\right\rangle>0$. For $m \geq 1$, it follows that

where

$$
\begin{gathered}
\mathscr{T}^{n} A_{m}=\left\{-m \leq x \leq m,-1 \leq y \leq g_{n}(x)\right\}, \\
\mathscr{T}^{n} A_{m}^{\prime}=\left\{-m \leq x \leq m, y \leq g_{n}(x)\right\},
\end{gathered}
$$

$$
g_{n}(x)=\max \left\{y:(x, y) \in \mathscr{T}^{n} A_{m}^{\prime}\right\} .
$$

Each $g_{n}$ is a concave real-valued function by Proposition $\mathrm{Al}(\mathrm{b})$, and that its maximum occurs at $x=0$ by symmetry. Also, the "ends" $(m,-1)$ and $(m, 1)$ are pinned, i.e., $g_{n}(-m)=g_{n}(m)=1$ for all $n$. Introduce the hitting times for $j \geq 0$ given by $t_{j}=\min \left\{n \geq 0: g_{n}(0) \geq j\right\}$. Consider the evolution of $\mathscr{T}^{n} A_{m}^{\prime}$ until time $t_{j+k}$. Let us compare with the process obtained by adding extra sites at time $t_{j}$ to fill $\left\{-m \leq x \leq m,-\infty \leq y \leq g_{t_{j}}(0)\right\}$, and then run until it hits level $j+k$. We conclude that $t_{j+k} \geq t_{j}+t_{k}-1(j, k \geq 0)$. Hence the sequence $\left(t_{j}-1 ; j \geq 0\right)$ is superadditive, and $\tau=\lim t_{j} / j=\sup \left(t_{j}-1\right) / j$ exists. Inverting, and noting that the limit set must be a subinterval of $[0,1] e_{2}$, it follows that (2.4) holds with $l_{m}=\tau^{-1}$.

Next we show that $l_{m} \rightarrow 1$ as $m \rightarrow \infty$. Thanks to Rick Durrett for suggesting the following construction of a shape that moves up with positive speed. Consider the function $f_{m}:(-m, m) \rightarrow \mathbb{R}$ given by

$$
f_{m}(x)=-\frac{m^{2}}{(m-x)(m+x)} .
$$

We claim there is an $m_{0}<\infty$ such that for $m \geq m_{0}$ and $0 \leq x_{0} \leq x \leq$ $\min \left\{x_{0}+1, m\right\}$,

$$
\max \left\{f_{m}(x), f_{m}\left(x_{0}\right)-2\right\} \geq f_{m}\left(x_{0}\right)+\left(x-x_{0}\right) f_{m}^{\prime}\left(x_{0}\right)-\frac{10}{\sqrt{m}} .
$$


(One can assume $f_{m}(x) \geq f_{m}\left(x_{0}\right)-2$ : otherwise, since $f_{m}^{\prime} \leq 0$, for each $x_{0}$ the left side is constant while the right side decreases. If $x_{0} \leq m-\sqrt{m}$, explicit calculation of the derivative then shows that $\Delta:=f_{m}(x)-f_{m}\left(x_{0}\right)-$ $\left(x-x_{0}\right) f_{m}^{\prime}\left(x_{0}\right) \geq-3 /(\sqrt{m}-1)$, which suffices for large $m$. If $x_{0} \geq m-\sqrt{m}$, then concavity of $f_{m}$ implies that $x-x_{0} \leq-2 / f_{m}^{\prime}\left(x_{0}\right) \leq 5\left(m-x_{0}\right)^{2} / m(m$ large). In this case, straightforward estimation yields $\Delta \geq-10\left(m-x_{0}\right) / m \geq$ $-10 / \sqrt{m}$ ( $m$ large), and the claim follows.) Now consider $\widehat{A}_{m}=\{-m \leq$ $\left.x \leq m,-\infty \leq y \leq f_{m}(x)\right\}$. Since $w(v) /\left\langle v, e_{2}\right\rangle=1$ for every $v \in \mathscr{S}$ such that $\left\langle v, e_{2}\right\rangle>0,(2.5)$ implies that for any $\varepsilon>0$ and $m$ sufficiently large, $n(1-\varepsilon) e_{2}+\widehat{A}_{m} \subset \mathscr{T}^{n} \widehat{A}_{m}$. Hence, by monotonicity, $[0,-1+n(1-\varepsilon)] e_{2} \subset \mathscr{T}^{n} A_{m}$ for all $n$, so $l_{m} \geq 1-\varepsilon$ as desired.

To check that $l_{m}<1$ for each $m$, let $\widetilde{A}_{m}=\left\{-m \leq x \leq m, y \leq f_{2 m}(x)\right\}$. Using compactness we can find a $\delta>0$ so that every $(x, y)$ with $x \in[-m, m]$ and $y=f_{2 m}(x)+1$ satisfies $\left|((x, y)+\mathcal{N}) \cap \widetilde{A}_{m}\right|<2-\delta$. The fact that $f_{2 m}(m)=-\frac{4}{3}$, monotonicity, and another application of compactness yield

$$
\mathscr{T}^{n} A_{m}^{\prime} \subset \mathscr{T}^{n}\left(\frac{7}{3} e_{2}+\tilde{A}_{m}\right) \subset\left(n[0,1-2 \varepsilon] e_{2}\right)^{\varepsilon}
$$

for a suitable choice of $\varepsilon>0$ and all sufficiently large $n$.

Example 5. Now take $d=2, \mathscr{N}=\left(\left(B(1)+e_{1}\right) \cup\left(B(1)-e_{1}\right)\right) \cap\left(B_{\infty}(1)-e_{2}\right)$, and $\theta=\frac{\pi}{4}=\frac{1}{2}|\mathcal{N}|$. In this case $L=K_{1 / w}^{*}=\{0\}$ but if $R$ is sufficiently large and $B(R) \subset A$, then

$$
\bigcup_{n \geq 0} \mathscr{T}^{n} A \text { is an unbounded set, }
$$

i.e., the occupied set exhibits sublinear growth. Complete trivality of $L$ follows from Proposition 6 and the fact that

$$
\lim _{v \rightarrow e_{2}} \frac{w(v)}{\left\langle v, e_{2}\right\rangle}=0
$$

To establish sublinear growth, introduce the modification $\widetilde{\mathscr{T}}$ of $\mathscr{T}$ that maps open sets into open sets:

$$
\widetilde{\mathscr{T}} A=\{(x, y):|A \cap((x, y)+\mathscr{N})|>\theta\} \cup A .
$$

Take $A=(-2,2) \times(-\infty, 1)$. It clearly suffices to show that

$$
B:=\bigcup_{n \geq 0} \widetilde{\mathscr{T}}^{n} A=(-2,2) \times(-\infty, \infty) .
$$

If $G$ is any set such that $G-\lambda e_{2} \subset G$ for all $\lambda \geq 0$, then $\widetilde{\mathscr{T}} G$ has the same property. Hence $B$ also has the same property and there is an upper semicontinuous $f:(-2,2) \rightarrow(0, \infty)$, symmetric about 0 , such that $B=$ $\{(x, y):-2<x<2, y<f(x)\}$. We claim that $f \equiv \infty$.

Assume not. If $f(x)<\infty$, then $|B \cap((x, f(x))+\mathcal{N})| \leq \frac{1}{2}|\mathcal{N}|$. Choose $x_{k} \in(-2,2)$ so that $x_{k} \rightarrow x_{0}$ and $f\left(x_{k}\right) \rightarrow \inf f$. If $x_{0} \in(-2,2)$, then clearly $\left|B \cap\left(\left(x_{0}, f\left(x_{0}\right)\right)+\mathscr{N}\right)\right|>\frac{1}{2}|\mathcal{N}|$. Hence the same must be true for $x_{k}$ when $k$ is large, a contradiction. We conclude that inf $f$ is achieved at the two boundary points. 
Introduce $g:(-2,2) \rightarrow(0, \infty]$ defined by $g(x)=\inf \{f(y): y \in[0, x)\}$ for $x \geq 0$, with $g(-x)=g(x)$. Also define $g_{1}$ by

$$
\widetilde{\mathscr{T}}(\{-2<x<2, y<g(x)\})=\left\{-2<x<2, y<g_{1}(x)\right\} .
$$

It is easy to see that $g(x)<g_{1}(x)$ provided $g(x)<\infty$ and

$$
\liminf _{h \rightarrow 0} \frac{g(x+h)-g(x)}{h}>-\infty .
$$

Since $g(2-)<\infty$ and $g$ is nonincreasing in $(0,2), g\left(x_{0}\right)<g_{1}\left(x_{0}\right)$ for some $x_{0} \in(0,2)$. However, for each $x$ there are two possibilities: either $f(x)=g(x)$, or there exists an $\varepsilon>0$ such that $\left.g\right|_{(x-\varepsilon, x+\varepsilon)} \equiv g(x)$. If $x_{0}$ falls in the first category then $f\left(x_{0}\right)<g_{1}\left(x_{0}\right)$, contradicting the definition of $f$. Otherwise, replace $x_{0}$ by $x_{0}^{\prime}:=$ the left endpoint of the interval containing $x_{0}$ on which $g$ is constant. Then $g\left(x_{0}^{\prime}\right)=f\left(x_{0}^{\prime}\right)$ and (2.7) must be satisfied at $x_{0}^{\prime}$. This forces $f\left(x_{0}^{\prime}\right)=g\left(x_{0}^{\prime}\right)<g_{1}\left(x_{0}^{\prime}\right)$, again a contradiction. Hence $f \equiv \infty$ and (2.6) is proved. In fact, one can show tht $\mathscr{T}^{n} A$ has height of order $\sqrt{n}$ in this example; any other sublinear power law can also be obtained by modifying $\mathscr{N}$ suitably.

Our final example identifies a class of three-dimensional neighbor sets for which critical threshold growth dynamics are convex-confined even though $K_{1 / w}^{*}$ $\neq\{0\}$. This indisputable counterexample to our main formula (1.15) indicates some of the additional complications when $d>2$.

Example 6. Let $d=3$. Suppose $\mathscr{N}=\left(\mathscr{N}^{\prime} \times[-1,0]\right)$, where $\mathscr{N}^{\prime} \in \mathscr{B}\left(\mathbb{R}^{2}\right)$ is a symmetric set with 0 in its interior. If $\theta=\frac{1}{2}|\mathcal{N}|$, then $K_{1 / w}^{*}=\left[0, \frac{1}{2}\right] e_{3}$, but we claim that $\mathscr{T}$ is convex-confined. The computation of the polar is routine. To show confinement it suffices to let $B_{R}$ denote an open two-dimensional (circular) disk of radius $R$ centered at the origin, and consider the dynamics starting from $A=B_{R} \times[0,1], R<\infty$. The key observation is simple enough: since each half-plane orthogonal to the $x y$-plane cuts $\mathscr{N}$ in half, there is an $\varepsilon>0$ such that

$$
\mathscr{T}^{n} A \subset\left(B_{R} \times[0,1]\right) \cup\left(B_{R-\varepsilon} \times[1, \infty]\right) \text { for all } n .
$$

Iterating, we get

$$
\mathscr{T}^{n} A \subset \bigcup_{k=0}^{\lfloor R / \varepsilon\rfloor}\left(B_{R-k \varepsilon} \times[k, k+1]\right) \text { for all } n .
$$

\section{The CASE $\theta>\frac{1}{2}|\mathcal{N}|$ AND THE UPPER Bound (1.18)}

Let us now begin the proof of Theorem 1. The reader might want to consult the Appendix at this point for a self-contained treatment of the formal set-up, basic notation, and various technicalities. Alternatively, these details can be consulted as they are needed.

To warm up, we first show that for $d \geq 2$, if the threshold exceeds one-half the volume of the neighbor set, then $\mathscr{T}$ is convex-confined: to each bounded set $A \in \mathscr{B}\left(\mathbb{R}^{d}\right)$ there corresponds an $R<\infty$ such that $\mathscr{T}^{n} A \subset B(R)$ for all $n \geq 0$. Observe the difference from the (asymmetric) one-dimensional case $\theta>\frac{1}{2}|\mathscr{N}|$, 
sketched in $\S 1$, where (one-sided) growth is possible. Note also that convexconfinement does not say our process stops in the sense that $\mathscr{T}^{n+1} A=\mathscr{T}^{n} A$ for some $n$, but only asserts that the limit of iterates is bounded.

Proposition 1. If $\theta>\frac{1}{2}|\mathcal{N}|$, then $\mathscr{T}$ is convex-confined.

Proof. Fix distinct coordinates $i, j \in\{1, \ldots, d\}$, and identify the linear span of $\left\{e_{i}, e_{j}\right\}$ with $\mathbb{R}^{2}$. Let $P_{i j}$ be the orthogonal projection of $\mathbb{R}^{d}$ onto this subspace. For any $u \in \mathscr{S}^{d-1},\left|\mathscr{N} \cap H_{u}^{-}\right|<\frac{1}{2}|\mathcal{N}|$ iff $\left|\mathcal{N} \cap H_{-u}^{-}\right|>\frac{1}{2}|\mathcal{N}|$. Since the map $\varphi \mapsto\left|\mathcal{N} \cap H_{(\cos \varphi) e_{i}+(\sin \varphi) e_{j}}^{-}\right|$is continuous, we can choose $u \in \mathscr{S}^{1} \subset$ $\mathscr{S}^{d-1}$ so that

$$
\left|\mathcal{N} \cap H_{u}^{-}\right|=\frac{1}{2}|\mathcal{N}|
$$

By the hypothesis, (3.1) and (1.11), $w(u)=w(-u)=0$. Continuity also implies that $w=0$ in a neighborhood of $u$ and $-u$, so we can find $v \neq u$ : $w(v)=w(-u)=0$ as well. Now choose $b_{i j}, c_{i j}$ large enough that

$$
A \subset c_{i j} \cdot\left(H_{u}^{-} \cap H_{-u}^{-} \cap H_{v}^{-} \cap H_{-v}^{-}\right) \subset\left\{x \in \mathbb{R}^{d}:\left\|P_{i j} x\right\|_{\infty} \leq b_{i j}\right\} .
$$

Since each of the half-spaces above has speed 0 and $A$ is enclosed within their intersection, monotonicity of $\mathscr{T}$ implies that $\left\|P_{i j}\left(\mathscr{T}^{n} A\right)\right\|_{\infty} \leq b_{i j}$ for all $n$. Set $b=\max \left\{b_{i j} ; 1 \leq i, j \leq d\right\}$ to deduce that $\left\|\mathscr{T}^{n} A\right\|_{\infty} \leq b$ for all $n$, proving convex confinement.

In the preceding proof $1 / w=\infty$ on $\{u,-u, v,-v\}$, so property (A.6) shows that $P_{i j}\left(K_{1 / w}^{*}\right)$ contains only the two-dimensional origin for each $i, j$. Hence $K_{1 / w}^{*}=\{\boldsymbol{0}\}$. Thus the case when $\theta>\frac{1}{2}|\mathcal{N}|$ is completely degenerate, and (1.8) and (1.15) hold starting from any bounded $A$. Let us now show in full generality that $K_{1 / w}^{*}$ provides an upper bound for the limit set $L$ of Euclidean threshold growth dynamics.

Proposition 2. Given any $\varepsilon>0$ and bounded set $A \in \mathscr{B}\left(\mathbb{R}^{d}\right)$, there is an $n_{0}<\infty$ such that

for all $n \geq n_{0}$.

$$
n^{-1} \mathscr{T}^{n} A \subset\left(K_{1 / w}^{*}\right)^{\varepsilon}
$$

Proof. For large $R<\infty$, define approximations to $K_{1 / w}, w$, and $K_{1 / w}^{*}$ by

$$
M_{R}=K_{1 / w} \cap B(R), \quad w_{R}=1 / \alpha_{M_{R}}, \quad L_{R}=M_{R}^{*},
$$

respectively. $w$ is bounded because $\theta>0$ and $\mathscr{N}$ has finite measure. So $M_{R}$ is bounded and contains a neighborhood of $\mathbf{0}$. The same holds for $L_{R}$ by Proposition A2(a)(ii)-(iii). We claim that for each $r>0$,

$$
\mathscr{T}\left(r L_{R}\right) \subset(r+1) L_{R} .
$$

To prove (3.2), suppose that $\alpha_{L_{R}}$ is differentiable in direction $u \in \mathscr{S}$, i.e., $\nu(u)=\nu_{L_{R}}(u)$ exists. Then monotonicity of $\mathscr{T},(1.11)$, and the fact that $w \leq w_{R}$ give

$$
\begin{aligned}
\mathscr{T}\left(r L_{R}\right) & \subset \mathscr{T}\left(r \alpha_{L_{R}}(u) \cdot u+H_{\nu(u)}^{-}\right) \\
& =r \alpha_{L_{R}}(u) \cdot u+w(\nu(u)) \cdot \nu(u)+H_{\nu(u)}^{-} \\
& \subset r \alpha_{L_{R}}(u) \cdot u+w_{R}(\nu(u)) \cdot \nu(u)+H_{\nu(u)}^{-} .
\end{aligned}
$$


The extent of the last set in direction $u$ is

$$
r \alpha_{L_{R}}(u)+\frac{w_{R}(\nu(u))}{\langle\nu(u), u\rangle}=(r+1) \alpha_{L_{R}}(u) .
$$

Differentiable directions $u$ are dense in $\mathscr{S}$ by Proposition A2(e), so (3.2) follows.

Proposition $\mathrm{A} 2(\mathrm{~g})(\mathrm{i})$ implies that $L_{R} \stackrel{H}{\rightarrow} K_{1 / w}^{*}$, so choose $R$ large enough that $L_{R} \subset\left(K_{1 / w}^{*}\right)^{\varepsilon / 2}$. Then, using the fact that $L_{R}$ contains a neighborhood of 0 and is bounded, choose $r$ large enough that $A \subset r L_{R}$ and $L_{R} \subset r \cdot \frac{\varepsilon}{2} \cdot B(1)$. For $n \geq r^{2}$, applying (3.2) one gets

$$
\begin{aligned}
n^{-1} \mathscr{T}^{n} A & \subset n^{-1} \mathscr{T}^{n}\left(r L_{R}\right) \subset \frac{n+r}{n} L_{R} \\
& \subset L_{R}+\frac{\varepsilon}{2} \cdot B(1) \subset\left(K_{1 / w}^{*}\right)^{\varepsilon / 2}+\frac{\varepsilon}{2} \cdot B(1)=\left(K_{1 / w}^{*}\right)^{\varepsilon},
\end{aligned}
$$

as desired.

\section{The MAIN Result: LOWeR BOUND (1.19)}

In this section we derive lower bound (1.19) for $\mathscr{T}$ under nondegeneracy assumption (1.16); in light of Proposition 2 this will complete the proof of Theorem 1. Our proof of the lower bound constitutes the major technical effort of the paper; it will be broken up into a series of lemmas. The first step expresses a simple idea that was mentioned in the Introduction: given any convex neighborhood $A$ of the origin, with smooth boundary in a vicinity of the direction $u \in \mathscr{S}$, the boundary of a sufficiently large multiple of $A$ is locally flat in direction $u$. Roughly, this means that a sizable chunk of the half-space $(r-\varepsilon) \alpha_{A}(u) \cdot u+H_{\nu_{A}(u)}^{-}$remains within $r A$. The precise formulation of the approximation lemma we need is as follows. Figure 5 is intended to help explain the gist of displays (4.1)-(4.3).

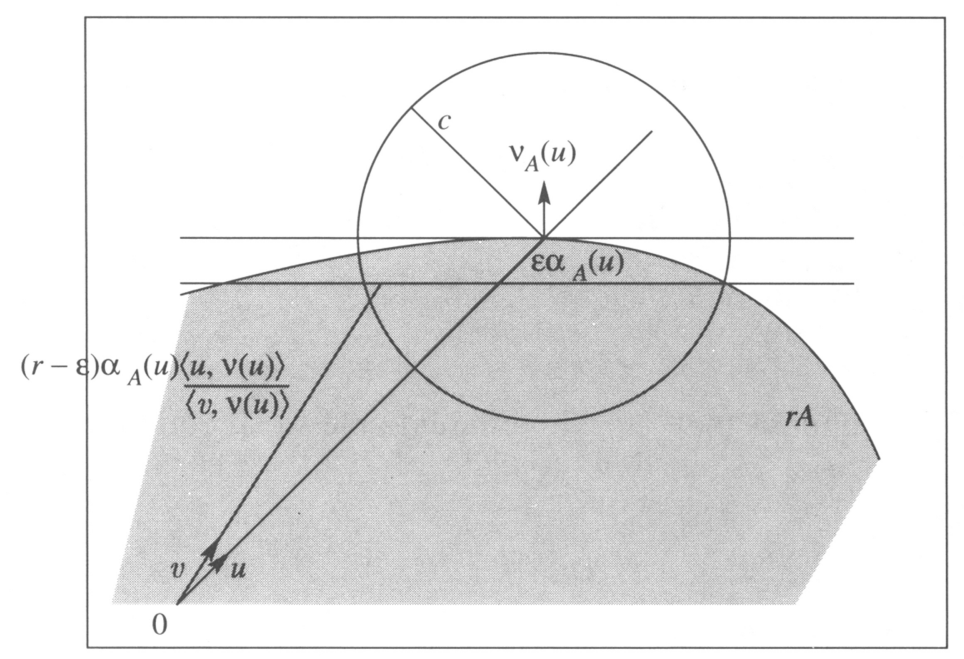

Figure 5 
Lemma 1. Assume that $A \subset \mathbb{R}^{d}$ is a bounded convex set with $\mathbf{0}$ in its interior, and that $\alpha_{A}$ is $\mathscr{C}^{1}$ on an open set $\mathscr{S}_{\mathrm{op}} \subset \mathscr{S}=\mathscr{S}^{d-1}$. Let $\mathscr{S}_{\mathrm{cl}}$ be a closed subset of $\mathscr{S}_{\mathrm{op}}$. Then for every $\varepsilon>0$ and $c<\infty$, there exists an $r_{0}<\infty$ such that for every $r \geq r_{0}$ and every $u \in \mathscr{S}_{\mathrm{cl}}$,

$$
\left((r-\varepsilon) \alpha_{A}(u) \cdot u+H_{\nu_{A}(u)}^{-}\right) \cap B\left(r \alpha_{A}(u) \cdot u, c\right) \subset r A \cap B\left(r \alpha_{A}(u) \cdot u, c\right) .
$$

Proof. We need to check that whenever $v$ satisfies

$$
\left\|r \alpha_{A}(v) v-r \alpha_{A}(u) u\right\| \leq c,
$$

it follows that

$$
(r-\varepsilon) \alpha_{A}(u) \frac{\left\langle u, \nu_{A}(u)\right\rangle}{\left\langle v, \nu_{A}(u)\right\rangle} \leq r \alpha_{A}(v) .
$$

By the triangle inequality and (4.2),

$$
r \alpha_{A}(v)\|v-u\| \leq c+r\left|\alpha_{A}(v)-\alpha_{A}(u)\right| .
$$

Also

$$
\begin{aligned}
\left(\frac{c}{r}\right)^{2} & \geq\left\|\alpha_{A}(v) v-\alpha_{A}(u) u\right\|^{2}=\left(\alpha_{A}(v)-\alpha_{A}(u)\right)^{2}+2 \alpha_{A}(u) \alpha_{A}(v)(1-\langle u, v\rangle) \\
& \geq\left(\alpha_{A}(v)-\alpha_{A}(u)\right)^{2} .
\end{aligned}
$$

Combine the last two inequalities to see that

$$
\|v-u\| \leq \frac{2 c}{r \min \left\{\alpha_{A}(u)\right\}}=\frac{\bar{c}}{r} .
$$

Since $\alpha_{A}$ is $\mathscr{C}^{1}$ on $\mathscr{S}_{\text {op }}$, for $u, v \in \mathscr{S}_{\text {cl }}$ we have

$$
\alpha_{A}(v)=\alpha_{A}(u)\left(1-\frac{\left\langle v-u, \nu_{A}(u)\right\rangle}{\left\langle v, \nu_{A}(u)\right\rangle}\right)+o(\|v-u\|) .
$$

Given $\delta>0$, choose $r_{0}=r_{0}(\delta)$ large enough that the $o$ term in this Taylor expansion is at most $\delta\|v-u\|$ when (4.2) holds, and $r \geq r_{0}$. Now (4.3) follows provided that

$$
\delta \leq \frac{\alpha_{A}(u)}{\bar{c}} \frac{\left\langle u, \nu_{A}(u)\right\rangle}{\left\langle v, \nu_{A}(u)\right\rangle} \varepsilon
$$

The numerators on the right are bounded below and the denominators are bounded above, so one can select a suitable $\delta=\delta(\varepsilon)$.

Lower bound (1.19) is easiest to verify when $\mathscr{N}$ is bounded and half-planes propagate in all directions. Our strategy is to find a good smooth approximation $L_{R}$ to $K_{1 / w}^{*}$ from the inside, and then show that a sufficiently large multiple $r L_{R}$ stays close to $r K_{1 / w}^{*}$ as transformation $\mathscr{T}$ is applied repeatedly. For the sake of clarity, we prove this special case of Theorem 1 before proceeding to consider the general situation.

Lemma 2. Conclusion (1.17) of Theorem 1 holds provided that $\mathscr{N}$ is bounded and $w>0$.

Proof. For a given large $R<\infty$, define approximations to $K_{1 / w}, w$, and $K_{1 / w}^{*}$ by

$$
M_{R}=\bigcap\left\{B(x, R): K_{1 / w} \subset B(x, R)\right\}, \quad w_{R}=1 / \alpha_{M_{R}}, \quad L_{R}=M_{R}^{*},
$$


respectively. It is not hard to check that $M_{R}$ is closed and strictly convex. Also, $w_{R} \leq w$, and $M_{R}$ converges to $\overline{\mathrm{co}}\left(K_{1 / w}\right)$ as $R \rightarrow \infty$. Hence, given $\varepsilon \in(0,1)$, by Proposition A2(g)(ii) we can choose $R$ so that

$$
(1-\varepsilon) K_{1 / w}^{*} \subset L_{R}
$$
by

If $A$ is star-shaped with $\mathscr{C}^{1}$ boundary, define auxiliary transformations $\mathscr{T}_{R}$

$$
\alpha_{\mathscr{T}_{R}(A)}(u)=\alpha_{A}(u)+\frac{w_{R}\left(\nu_{A}(u)\right)}{\left\langle u, \nu_{A}(u)\right\rangle} .
$$

(Roughly, $\mathscr{T}_{R}$ acts like $\mathscr{T}$ but uses $w_{R}$ instead of $w$ and pretends that $A$ has flat boundary in the direction of each $u$.) By (A.4) and the fact that $M_{R}=L_{R}^{*}$,

$$
\mathscr{T}_{R}\left(r L_{R}\right)=(r+1) L_{R} \text {. }
$$

Choose $c<\infty$ so that $\mathscr{N} \subset B(c)$, and then apply Lemma 1 to pick $r_{0}$ large enough that (4.1) holds for $A=r L_{R}, r \geq r_{0}$. Under $\mathscr{T}$, sites outside $A$ in direction $u$ encounter an occupied set that effectively covers $(r-\varepsilon) \alpha_{A}(u) \cdot u+$ $H_{\nu_{A}(u)}^{-}$. Thus $\mathscr{T}$ extends $A$ in direction $u$ to at least

$$
\left((r-\varepsilon) \alpha_{A}(u)+\frac{w\left(\nu_{A}(u)\right)}{\left\langle u, \nu_{A}(u)\right\rangle}\right) \cdot u
$$

Since $w_{R} \leq w$, it follows that for $r \geq r_{0}$,

$$
\mathscr{T}_{R}\left((r-\varepsilon) L_{R}\right) \subset \mathscr{T}\left(r L_{R}\right) \text {. }
$$

Iterating (4.5) and (4.6), if $n \geq 1$ then

$$
\left(r_{0}+(1-\varepsilon) n\right) L_{R} \subset \mathscr{T}^{n}\left(r_{0} L_{R}\right) .
$$

Large balls are locally flat so for sufficiently large $m, B\left(m+\frac{1}{2} \min w\right) \subset$ $\mathscr{T} B(m)$. Hence if $A$ contains a large ball $B(m)$, there exists a finite $n_{0}$ such that $r_{0} L_{R} \subset \mathscr{T}^{n_{0}} A$. For each $n \geq n_{0} / \varepsilon$, monotonicity of $\mathscr{T},(4.7)$ and (4.4) yield

$$
\begin{aligned}
\mathscr{T}^{n} A & \supset \mathscr{T}^{n-n_{0}}\left(r_{0} L_{R}\right) \supset(1-\varepsilon)\left(n-n_{0}\right) L_{R} \\
& \supset(1-\varepsilon)^{2}\left(n-n_{0}\right) K_{1 / w}^{*} \supset n(1-3 \varepsilon) K_{1 / w}^{*} .
\end{aligned}
$$

Divide by $n$ to complete the proof of (1.19) and the lemma.

Note that we have now established the limiting shape formula (1.15) whenever $\mathscr{N}$ is symmetric (i.e., $-\mathscr{N}=\mathscr{N}$ ): if $\theta<\frac{1}{2}|\mathcal{N}|$ then $w>0$ and Lemma 2 applies, whereas if $\theta \geq \frac{1}{2}|\mathcal{N}|$ then $w \equiv 0$ and $\mathscr{T}$ is convex-confined. The next step in our demonstration of Theorem 1 is to assume that $w$ is not strictly positive, but that (1.16) holds for some $u$. Without loss of generality, assume $u=-e_{1}$. A new construction of the inner $R$-approximants $M_{R}, w_{R}$, and $L_{R}$ is needed to handle this case.

Lemma 3. Conclusion (1.17) of Theorem 1 holds provided $\mathcal{N}$ is bounded, $w>0$ on $H_{-e_{1}}^{-} \cap \mathscr{S}$, and $w(u)=0$ for some $u \in H_{e_{1}}^{-} \cap \mathscr{S}$.

Proof. Fix a large $R>0$ and define the set $W_{R}$ by its boundary function

$$
\alpha_{W_{R}}(u)= \begin{cases}\infty & \text { if }\|v-u\|<1 / R \text { for some } v \text { such that } w(v)=0 \\ 1 / w(u) & \text { otherwise. }\end{cases}
$$


Also, for $0<b<1$, write

$$
g_{b}(x)= \begin{cases}\sqrt{1-x^{2}}, & x \in[-b, b], \\ \frac{1-b|x|}{\sqrt{1-b^{2}}}-\frac{1}{R^{2}} \frac{(|x|-b)^{2}}{|x|-b+1} & \text { otherwise. }\end{cases}
$$

Note that $g_{b}: \mathbb{R} \rightarrow(-\infty, 1]$ is strictly convex. Hence the sets

$$
G_{R, b}=\left\{x \in \mathbb{R}^{d}: x_{1} \leq R g_{b}\left(\frac{1}{R} \sqrt{x_{2}^{2}+\cdots+x_{d}^{2}}\right)\right\}
$$

are also strictly convex. Let $b>0$ be any number such that $W_{R} \subset \operatorname{Re}_{1}+G_{R, b}$; hypothesis (1.16) guarantees the existence of such a $b$. An $R$-approximating region is any set of the form $D=x+U G_{R, b}$, where $x \in B(R), U$ is an orthogonal linear map, and $W_{R} \subset D$. Now define

$$
M_{R}=\bigcap\{D: D \text { is an } R \text {-approximating region }\} .
$$

Then $M_{R}$ is strictly convex, and $M_{R} \downarrow \overline{\mathrm{co}}\left(K_{1 / w}\right)$ as $R \rightarrow \infty$. As in the previous lemma, let $w_{R}=1 / \alpha_{M_{R}}$ and $L_{R}=M_{R}^{*}$. Also, if $f$ is a nonnegative $\mathscr{C}^{1}$ function defined on $\operatorname{in}\left(\left\{u: \alpha_{L_{R}}(u)>0\right\}\right)$, set $f$ equal to 0 elsewhere, and define the auxiliary transformation $\mathscr{T}_{R}$ by

$$
\alpha_{\mathscr{F}_{R}\left(K_{f}\right)}(u)= \begin{cases}f(u)+\frac{w_{R}\left(\nu_{K_{f}}(u)\right)}{\left\langle u, \nu_{K_{f}}(u)\right\rangle}, & f(u)>0 \\ 0, & f(u)=0 .\end{cases}
$$

Again, for any $r>0, \mathscr{T}_{R}\left(r L_{R}\right)=(r+1) L_{R}$. By Proposition A2(g)(ii), $L_{R} \stackrel{H}{\rightarrow}$ $K_{1 / w}^{*}$; choose $R$ large enough that $(1-\varepsilon) K_{1 / w} \subset L_{R}$. Also, by strict convexity of $L_{R}$, if we fix $a>0$ so that $a e_{1} \in \operatorname{in}\left(L_{R}\right)$, then Proposition A2(f)(iii) implies that $\alpha_{L_{R}-a e_{1}}$ is $\mathscr{C}^{1}$ on $\mathscr{S} \backslash\left\{-e_{1}\right\}$. Applying Lemma 1 to $r\left(L_{R}-\varepsilon a e_{1}\right)$, we can find an $r_{0}$ so that for $r \geq r_{0}$,

$$
\begin{aligned}
\mathscr{T}\left(r L_{R}\right) & =r a e_{1}+\mathscr{T}\left(r\left(L_{R}-a e_{1}\right)\right) \\
& \supset r a e_{1}+\mathscr{T}_{R}\left((r-\varepsilon)\left(L_{R}-a e_{1}\right)\right) \\
& =\varepsilon a e_{1}+(r+1-\varepsilon) L_{R} .
\end{aligned}
$$

At first glance, (4.8) would appear to finish the proof. We have shown that starting from $A=r_{0} L_{R}$, with $R$ sufficiently large, the limit set for iterates of $\mathscr{T}$ is close to $L=K_{1 / w}^{*}$. But so far there is the possibility that larger and larger $A$ might be required for the dynamics to approach $L$, i.e., that $r_{0}$ might grow with $R$. To rule out this scenario we appeal to the following fact.

Claim. Let $\mathscr{S}_{c}$ be a closed subset of $\operatorname{in}\left(\mathscr{S} \cap H_{-e_{1}}^{-}\right)$such that in $\left(\mathscr{S}_{c}\right) \neq \varnothing$, and $K=[0, \infty) \cdot \mathscr{S}_{c} \subset \mathbb{R}^{d}$ is convex. Assume also that $\eta=\inf \{w(v): v \in \mathscr{S}$ and $\langle u, v\rangle=0$ for some $\left.u \in \mathscr{S}_{c}\right\}>0$. Then there is a bounded set $A_{0} \in \mathscr{B}\left(\mathbb{R}^{d}\right)$ such that the iterates of $\mathscr{T}$ starting from $A_{0}$ eventually cover any bounded Borel set $B \subset K$ (i.e., $B \subset \mathscr{T}^{n_{0}} A_{0}$ for some $n_{0}<\infty$ ).

Proof of the claim. It suffices to show the existence of a set $A_{0}$ and number $k$ so that $\mathscr{T}^{n} A_{0}$ eventually covers any compact subset of $k e_{1}+K$. The argument leading to (4.8) produces an $A_{0}$ and a convex closed set $\mathscr{S}^{\prime} \subset \mathscr{S}_{c}$ such that $\mathscr{T}^{n} A_{0}$ eventually covers every compact subset of $K^{\prime}=[0, \infty) \cdot \mathscr{S}^{\prime}$. We will prove that the same $A_{0}$ satisfies the claim. 
In order to apply Lemmas 1 and 2 , let us rephrase the problem slighty. Identify $\mathbb{R}^{d-1}$ with $\left\{x_{1}=0\right\}$, and define $V \subset \mathbb{R}^{d-1}$ by $e_{1}+V=[0, \infty) \mathscr{S}_{c} \cap\left(e_{1}+\right.$ $\left.\left\{x_{1}=0\right\}\right)$. So $[0, \infty) \mathscr{S}_{c}=[0, \infty)\left(e_{1}+V\right)$. Define $V^{\prime}$ analogously in terms of $\mathscr{S}^{\prime}$. Let $\eta^{\prime}>0$ be the minimal length of all projections from any $w(v) v$ to $\left\{x_{1}=0\right\}$ :

$$
\eta^{\prime}=\min \left\{w(v) \sqrt{\|v\|^{2}-\left\langle v, e_{1}\right\rangle^{2}}: v \in \mathscr{S} \text { and }\langle u, v\rangle=0 \text { for some } u \in \mathscr{S}\right\} .
$$

Now let $W$ be any compact subset of $V$ with nonempty interior and $\mathscr{C}^{1}$ boundary. Lemma 1 says the boundary of $r W$ is nearly flat for $r \geq r_{0}$, and so $\mathscr{T}\left([0, \infty)\left(e_{1}+W\right)\right) \supset[r, \infty)\left(e_{1}+\left(1+\frac{1}{2} \eta^{\prime}\right) W\right)$. Choose $m$ large enough that $\mathscr{N} \subset B(m / 2)$. It follows that $\mathscr{T}^{n}\left([0, \infty)\left(e_{1}+W\right)\right)$ covers $[r+(n-1) m, \infty)\left(e_{1}+\left(1+\frac{n}{2} \eta^{\prime}\right) W\right)$. Hence there is a small $\delta>0$ and a large $k_{0}<\infty$ such that $\mathscr{T}^{n}\left([0, \infty)\left(e_{1}+W\right)\right)$ eventually covers every compact subset of $k_{0} e_{1}+[0, \infty)\left(e_{1}+(1+2 \delta) W\right)$. Note that $\delta$ depends only on $m$ and $\eta^{\prime}$, but not on $W$. (On the other hand, $k_{0}$ does depend on $W$.)

If $W$ is convex but not differentiable, use the recipe of Lemma 2 to find a $\mathscr{C}^{1}$ convex $W \supset W^{\prime} \supset(1-\delta) W$. The discussion of the last paragraph thereby produces a large $k_{1}<\infty$ such that every compact subset of $k_{1} e_{1}+$ $[0, \infty)\left(e_{1}+(1+\delta) W\right)$ is eventually included in $\mathscr{T}^{n}\left([0, \infty)\left(e_{1}+W\right)\right)$.

The final step in justifying our claim is to apply what we have just proved consecutively to $V^{\prime},(1+\delta) V^{\prime} \cap V,(1+2 \delta) V^{\prime} \cap V, \ldots$, noting that these sets reach $V$ in a finite number of steps.

To complete the proof of Lemma 3, note that any closed subset of in $\left(\left\{u: \alpha_{L_{R}}(u)>0\right\}\right)$ satisfies the hypotheses of the claim by Proposition A2(b). Let $A$ be any initial set that includes $A_{0}$ of the claim. For the chosen $R$ and $r_{0}$ in (4.8), select $n_{0}$ large enough that $r_{0} L_{R} \subset \mathscr{T}^{n_{0}} A$. Then for $n>n_{0} / \varepsilon$,

$$
\begin{aligned}
\mathscr{T}^{n} A & \supset \mathscr{T}^{n-n_{0}}\left(r_{0} L_{R}\right) \supset\left(n-n_{0}\right) \varepsilon a e_{1}+(1-\varepsilon)\left(n-n_{0}\right) L_{R} \\
& \supset\left(n-n_{0}\right) \varepsilon a e_{1}+(1-\varepsilon)^{2}\left(n-n_{0}\right) K_{1 / w}^{*} \\
& \supset n\left[\left(1-\frac{n_{0}}{n}\right) \varepsilon a e_{1}+(1-3 \varepsilon) K_{1 / w}^{*}\right],
\end{aligned}
$$

and (1.17) follows.

Finally, to finish the proof of Theorem 1, we remove the assumption that $\mathcal{N}$ is bounded.

Lemma 4. If (1.17) holds whenever $\mathscr{N}$ is bounded, then it holds for any $\mathcal{N}$ with finite volume.

Proof. The upper bound (1.18) holds for any $\mathscr{N}$ with finite volume since boundedness was not assumed in Proposition 2. To derive the lower bound (1.19), define $\mathscr{N}_{k}=\mathscr{N} \cap B(k)$ and let $w_{k}$ be the corresponding width function. Since $w_{k} \uparrow w<\infty$ as $k \rightarrow \infty$, and assumption (1.16) holds for $w$, it also holds for $w_{k}$ once $k$ is sufficiently large. Thus Lemma 3 applies to each $\mathscr{N}_{k}$, and so four facts from Proposition A2 yield the desired inclusion (1.19). First, by (g)(iii), $\overline{\operatorname{co}}\left(K_{1 / w_{k}}\right) \downarrow \overline{\operatorname{co}}\left(K_{1 / w}\right)$ as $k \rightarrow \infty$. Then (a) parts (i) and (iii) imply that $K_{1 / w_{k}}^{*}=\overline{\mathrm{co}}\left(K_{1 / w_{k}}\right)^{*}$ and $K_{1 / w}^{*}=\overline{\mathrm{co}}\left(K_{1 / w}\right)^{*}$. Finally, applying (g)(ii), $\left(n^{-1} \mathscr{T}^{n} A\right)^{\varepsilon} \supset K_{1 / w}^{*}$ eventually in $n$. 
Our limit theorem does not apply if the polar of $1 / w$ has a positive dimension less than $d$. We do not know any such cases for which the iterates of $\mathscr{F}$ fail to have a limiting shape or the limiting shape is not convex. Recall, however, Examples 4 and 6 from $\S 2$ : they show that the shape of $L$ need not agree with $K_{1 / w}^{*}$ in these partially degenerate cases.

\section{LATTICE THRESHOLD GROWTH DYNAMICS AND THE THRESHOLD-RANGE SCALING LIMIT}

Let us now return to cellular automaton rules of the form (1.6) and develop their connection with the limiting shapes $L$ of Theorem 1 . The first order of business is a corresponding shape result on $\mathbb{Z}^{d}$. In contrast to the Euclidean setting, it seems cumbersome to obtain an explicit formula for $L$ on the lattice. Instead, our next theorem exploits subaddivitity to establish an implicit characterization.

We introduce two rather ad hoc notions that incorporate technical requirements for our results on the lattice $\mathbb{Z}^{d}$. Say that $A \subset \mathbb{Z}^{d}$ is completely symmetric if it is invariant with respect to switching sign of the $i$ th coordinate and transposing the $i$ th and $j$ th coordinates $(i, j=1, \ldots, d)$. Call $A \subset \mathbb{Z}^{d}$ obese if $A$ is completely symmetric and the following two-part "cone condition" holds:

(i) If $x \in A$ and $x_{1}>0$, then $x-e_{1} \in A$,

(ii) If $x \in A$ and $x_{1}>x_{2}>0$, then $x-e_{1}+e_{2} \in A$.

Theorem 2. Assume that $N \subset \mathbb{Z}^{d}$ is obese, and $A$ is a finite set such that $T^{n} A \uparrow \mathbb{Z}^{d}$. Then as $n \rightarrow \infty$,

$$
n^{-1} T^{n} A \stackrel{H}{\rightarrow} L=L(N, \theta) \subset \mathbb{R}^{d},
$$

and $L$ is convex.

Proof. First pick $m, R$, and $n_{0}$ large enough that $N \subset B_{1}(m), B=B_{\infty}(R) \supset$ $A$, and $T^{n} B \supset B_{\infty}(R)$. For each $x \in \mathbb{R}^{d}$ define

$$
t_{x}=\min \left\{n \geq 0: a+B \subset T^{n} B \text { for every } a \in B_{\infty}(x, 1) \cap \mathbb{Z}^{d}\right\} .
$$

Fix $x, y \in \mathbb{R}^{d}$, and assume there is a $c \in \mathbb{Z}^{d}$ such that $\|c-(x+y)\|_{\infty} \leq 1$. Then there exist sites $a, b \in \mathbb{Z}^{d}$ such that $\|a-x\|_{\infty} \leq 1,\|b-y\|_{\infty} \leq 1$, and $c=a+b$. (This is easy to check for $d=1$, and the general case follows immediately.) As a consequence of this fact and monotonicity of $T$, the $t_{x}$ are subadditive, i.e., $t_{x+y} \leq t_{x}+t_{y}$ for all $x, y \in \mathbb{R}^{d}$. Consequently

$$
\tau(x):=\inf _{n \geq 1} \frac{t_{n x}}{n}=\lim _{n \rightarrow \infty} \frac{t_{n x}}{n} .
$$

Using the a priori bounds

$$
\frac{\|x\|_{1}}{m} \leq t_{x} \leq n_{0}\|x\|_{\infty}
$$

for real $\lambda$, with $n=\lfloor\lambda\rfloor$, we have

$$
\left|\frac{t_{\lambda x}}{\lambda}-\frac{t_{n x}}{n}\right| \leq \frac{2 n_{0}\|x\|_{\infty}}{n}
$$


Hence the limit in (5.2) extends to reals $\lambda \rightarrow \infty$, and $0<\tau(x)<\infty$ for every $x \neq 0$.

Now define $L=\{\tau \leq 1\}$. Since $\tau$ is a norm, $L$ is a closed convex bounded neighborhood of 0 . Pick $\varepsilon>0$; to obtain our shape result we need to show that

$$
(1-\varepsilon) L \subset n^{-1}\left(T^{n} B+B_{\infty}\left(\frac{1}{2}\right)\right) \subset(1+\varepsilon) L
$$

once $n$ is sufficiently large. For the left-hand inclusion, if $\tau(x) \leq 1-\varepsilon$ then $t_{n x}<n$ eventually in $n$, implying that $n x \in T^{n} B+B_{\infty}\left(\frac{1}{2}\right)$. For the right-hand inclusion we need to use the fact that $T^{n} B$ is obese, by Proposition $\mathrm{Al}(\mathrm{c})$. If an obese set $F$ is sufficiently large, then $z+B \subset T^{n_{0}} F$ whenever $y \in F$ and $z \in B_{\infty}(y, 1)$. So $n x \in T^{n} B$ implies that $t_{n x} \leq n+n_{0}$, and hence $\tau(x) \leq 1+n_{0} / n$. Therefore $x \in(1+\varepsilon) L$ as soon as $n \varepsilon \geq n_{0},(5.3)$ holds, and Theorem 2 is proved for $B$ instead of $A$. The final step should be familiar by now: since $T^{n} A \uparrow \mathbb{Z}^{d}$ by hypothesis, $B \subset T^{n_{1}} A$ for some $n_{1}$. Hence

$$
T^{n-n_{1}} B \subset T^{n} A \subset T^{n} B
$$

for $n \geq n_{1}$. Divide by $n$, then send $n \rightarrow \infty$ to complete the proof.

The reader may well ask how one checks whether any given threshold growth rule $T_{\theta, N}$ admits initial $A$ that eventually cover the entire lattice. For obese neighbor sets $N$ our next proposition states that if the threshold $\theta$ is small enough in comparison with $\# N$, then large $A$ satisfying the hypothesis of Theorem 1 always exist. For example this result applies to the range $10 \mathrm{CA}$ discussed in the Introduction, so we have established the limit shape $L$ suggested by Figure 2. We also present a lattice counterpart to Proposition 1, stating that the iterates of any $A$ under $T_{\theta, N}$ eventually stop if $N$ is obese and $\theta$ is large compared to \#N. Lattice complications preclude an exact evaluation of the cutoff, but as expected it occurs at $\theta \approx \frac{1}{2} \# N$ when $N$ is large.

Proposition 2. Let $N$ be obese.

(a) If $\theta \leq \frac{1}{2}\left[\# N-(1+2 \sqrt{d}) \#\left(N \cap\left\{x_{1}=0\right\}\right)\right]$, then there is a finite $A \subset \mathbb{Z}^{d}$ such that $T^{n} A \uparrow \mathbb{Z}^{d}$.

(b) If $\theta>\frac{1}{2}(\# N-1)$, then for each finite $A \subset \mathbb{Z}^{d}$ there exists an $n$ such that $T^{n+1} A=T^{n} A$.

Proof. Our argument generalizes one from the Appendix of [6]. Fix a site $x$ in the "lattice ring" $(B(R+1) \backslash B(R)) \cap \mathbb{Z}^{d}$. Both (a) and (b) are immediate once we show that for large enough $R$,

$$
\left.\frac{1}{2}[\# N-(1+2) \sqrt{d}) \#\left(N \cap\left\{x_{1}=0\right\}\right)\right] \leq \#(B(R) \cap(x+N)) \leq \frac{1}{2}(\# N-1) \text {. }
$$

Now if $y \in B(R) \cap(x+N)$, then $y=x+z$ for some $z \in N$, and hence

$$
\|y\|^{2}-\|x\|^{2}=2\langle x, z\rangle+\|z\|^{2} .
$$

Thus, if $y \in B(R)$ then $\langle x, z\rangle<0$. By symmetry, $2 \#\{z \in N:\langle x, z\rangle<0\}$ $+1 \leq \# N$, and the upper bound in (5.4) follows. On the other hand, $y \in B(R)$ provided that the following two conditions are satisfied for some $\varepsilon>0$ :

$$
\langle x, z\rangle \leq-(1+\varepsilon) R, \quad \text { and }\|z\|^{2} \leq 2 \varepsilon R-1 .
$$


Note that once $\varepsilon$ is chosen, the first inequality holds for all $z$ if we take $R$ sufficiently large. Dividing the second inequality by $\|x\|$, it therefore suffices to produce a lower bound on

$$
c=\operatorname{int}_{u \in \mathscr{S}} \#\{z \in N:\langle u, z\rangle \leq-(1+\varepsilon)\} .
$$

Fix $u \in \mathscr{S}$. Then $\left\langle u, e_{i}\right\rangle \geq 1 / \sqrt{d}$ for some $i$; without loss of generality assume $i=1$. For every $w \in \mathbb{Z}^{d-1} \cap\left\{x_{1}=0\right\}$, the one-dimensional lattice slice $w+\mathbb{Z} e_{1}$ intersects the set

$$
\#\left\{x \in \mathbb{R}^{d}:-(1+\varepsilon) \leq\langle u, x\rangle \leq(1+\varepsilon)\right\}
$$

in at most $1+2 \sqrt{d}$ points provided $\varepsilon<1 / 2 \sqrt{d}$. Now use obesity of $N$ to conclude that the cardinality of the intersection of set (5.5) with $N$ is a most $(1+2 \sqrt{d}) \#\left(N \cap\left\{x_{1}=0\right\}\right)$. Therefore

$$
2 c+(1+2 \sqrt{d}) \#\left(N \cap\left\{x_{1}=0\right\}\right) \geq \# N,
$$

which completes the proof.

As mentioned in our Introduction, the limit shape $L_{10}$ for the $\rho=10$, $\theta=147$ CA of Figure 2 is close to the limit $L$ for Euclidean threshold growth with $\mathscr{N}=B_{\infty}(1)$ and $\theta=\frac{4}{3}$. Thus the boundary of $L_{10}$ has a shape similar to the 7 th smallest ring in Figure 3. Let us now express this connection as a limit theorem for CA dynamics with large range. To do so, we first formalize the notion of lattice transformations $T_{k}$ converging to a Euclidean transformation $\mathscr{T}$ as $k \rightarrow \infty$.

Definition. A sequence of threshold growth CA rules $T_{k}=T_{\theta_{k}, N_{k}}, k \geq 1$, converges to the Euclidean threshold growth rule $\mathscr{T}=\mathscr{T}_{\theta}, \mathcal{N}$ iff

$$
\theta_{k} \rightarrow \infty \quad \text { and } \quad \frac{N_{k}}{\sqrt{\theta_{k}}} \stackrel{H}{\rightarrow} \frac{\mathscr{N}}{\sqrt{\theta}} \quad \text { as } k \rightarrow \infty .
$$

Theorem 3. Assume that $T_{k}$ converges to $\mathscr{T}$. Assume further that each $N_{k}$ is obese, and that $\theta<\frac{1}{2}|\mathcal{N}|<\infty$. Then limiting shapes $L_{k}$ exist for all sufficiently large $k$, and

$$
\frac{1}{\sqrt{\theta_{k}}} L_{k} \stackrel{H}{\rightarrow} \frac{1}{\sqrt{\theta}} L \quad \text { as } k \rightarrow \infty
$$

where $L=L_{\theta, \mathscr{N}}$ is the limiting shape for $\mathscr{T}_{\theta, \mathscr{N}}$.

Proof. Since $\mathscr{T}_{c^{2} \theta, c \mathscr{N}}(c A)=c \mathscr{T}_{\theta, \mathscr{N}} A, c>0$, we can assume without loss of generality that $\theta=1$. Let us start the proof with a few observations. First of all, $\mathscr{N}$ must be obese (the definition for continuous sets being analogous to that in $\mathbb{Z}^{d}$ ), and hence star-shaped. Secondly, for fixed $\varepsilon>0$ and large enough $k$,

$$
(1-\varepsilon) \mathscr{N} \subset \frac{1}{\sqrt{\theta_{k}}}\left(N_{k}+B_{\infty}\left(\frac{1}{2}\right)\right) \subset(1+\varepsilon) \mathcal{N} .
$$

The upper bound in (5.6) follows from the assumed convergence of neighbor sets, the fact that $\mathcal{N}$ is star-shaped and the fact that $\alpha_{\mathcal{N}}$ is bounded below. To verify the lower bound, assume that $x$ is in the leftmost set in (5.6) but not 
in the middle set. Let $F \subset \mathbb{R}^{d}$ denote the set of points with all coordinates nonnegative; we can assume tht $x \in F$. Then $x+F$ has empty intersection with the middle set of (5.6) by obesity of $N_{k}$. Since $\mathscr{N}$ is obese, there is an $\eta>0$ and $y \in \mathbb{R}^{d}$ such that $B(y, \eta) \subset(x+F) \cap \mathscr{N}$. Hence $y$ is at least distance $\eta$ from $\left(1 / \sqrt{\theta_{k}}\right) N_{k}$, a contradiction. Thirdly, as $k \rightarrow \infty$,

$$
\# N_{k} \sim \theta_{k}|\mathcal{N}| \text {, and } \#\left(N_{k} \cap\left\{x_{1}=0\right\}\right) \sim \sqrt{\theta_{k}} \sigma\left(\mathscr{N} \cap\left\{x_{1}=0\right\}\right) \text {. }
$$

( $\sigma$ denotes $d-1$ dimensional volume.) Lastly, since $|\mathcal{N}|>2$, Proposition 2 and Theorem 2 imply that $L_{k}$ exists for large $k$.

We are now prepared for the heart of the argument. Fix $\varepsilon>0$. Let $\mathscr{T}^{\prime}=$ $\mathscr{T}_{1,(1-\varepsilon) \mathcal{N}}, \mathscr{T}^{\prime \prime}=\mathscr{T}_{1,(1+\varepsilon) \mathscr{N}}$, and also introduce the rescaled lattice dynamics $\mathscr{T}_{k}, k \geq 1$, given by

$$
\mathscr{T}_{k} A=\frac{1}{\sqrt{\theta_{k}}}\left(T_{k}\left(\left(\sqrt{\theta_{k}} A\right) \cap \mathbb{Z}^{d}\right)+B_{\infty}\left(\frac{1}{2}\right)\right),
$$

for each $A \subset \mathbb{R}^{d}$. Starting from sufficiently large "cubes" $A_{k}=B_{\infty}\left(R_{k}\right)$, as $n \rightarrow \infty$ one gets

$$
n^{-1} \mathscr{T}_{k}^{n} A_{k} \stackrel{H}{\rightarrow} \mathscr{L}_{k}=\frac{1}{\sqrt{\theta_{k}}} L_{k}
$$

We claim next that if $k$ is large enough and $A$ is of the form $\left(A^{\#}+B_{\infty}\left(\frac{1}{2}\right)\right) / \sqrt{\theta_{k}}$ for some $A^{\#} \subset \mathbb{Z}^{d}$, then $\mathscr{T}^{\prime} A \subset \mathscr{T}_{k} \subset \mathscr{T}^{\prime \prime} A$. Assume first that $x \in \mathscr{T}^{\prime} A$. Let $x^{\prime} \in \mathbb{Z}^{d}$ be such that

$$
\left\|x-\frac{1}{\sqrt{\theta_{k}}} x^{\prime}\right\|_{\infty} \leq \frac{1}{2 \sqrt{\theta_{k}}} .
$$

Then if $\theta_{k}$ is small enough, all cubes

$$
\frac{1}{\sqrt{\theta_{k}}} B_{\infty}\left(y, \frac{1}{2}\right)
$$

with $y \in A^{\#}$ that have nonempty intersection with $x+(1-\varepsilon) \mathcal{N}$ are completely contained in $\left(x^{\prime}+N_{k}+B_{\infty}\left(\frac{1}{2}\right)\right) / \sqrt{\theta_{k}}$. The number of such cubes is at least $\theta_{k}$; otherwise $|x+(1-\varepsilon) \mathscr{N} \cap A|<\theta_{k}^{-1} \theta_{k}$, a contradiction. Hence $x^{\prime} \in \mathscr{T}_{k} A$, and consequently $x \in \mathscr{T}_{k} A$. Now assume that $x \in \mathscr{T}_{k} A$. Then there is an $x^{\prime \prime} \in \mathbb{Z}^{d}$ satisfying (5.7) and such that the number of cubes (5.8) with $y \in A^{\#}$ and completely inside $\left(x^{\prime \prime}+N_{k}+B_{\infty}\left(\frac{1}{2}\right)\right) / \sqrt{\theta_{k}}$ is at least $\theta_{k}$. If $k$ is large enough, then all these cubes are inside $x+(1+\varepsilon) \mathcal{N}$, so $|(x+(1+\varepsilon) \mathcal{N}) \cap A| \geq 1$, and hence $x \in \mathscr{T}^{\prime \prime} A$.

Passing to the limit, the claim we have just proved yields

$$
L_{1,(1-\varepsilon) \mathcal{N}} \subset \mathscr{L}_{k} \subset L_{1,(1+\varepsilon) \mathscr{N}}
$$

For every bounded Borel $\mathscr{N}, \mathscr{N}^{\prime}$ and $A, \mathscr{T}_{\theta, \mathcal{N}^{\prime}} A \subset \mathscr{T}_{\theta-\left|\mathcal{N}^{\prime} \backslash \mathcal{N}\right|, \mathcal{N}^{\prime}} A$. To complete the proof of the theorem, it therefore suffices to verify that $L_{\theta, \mathcal{N}}$ converges to $L_{1, \mathcal{N}}$ if $\theta$ increases or decreases to 1 . But this follows directly from Proposition A2(g).

Theorem 3 establishes that the asymptotic shape of a threshold growth CA with large range is well approximated by a more explicit Euclidean threshold 
growth limit $L$. In this sense the continuous process is more tractable. Suppose, however, that we want to study detailed aspects of the geometry of $\mathscr{T}^{n} A$, e.g., boundary behavior or interaction effects between several growing regions. One approach is to visualize the Euclidean dynamics on a computer. Apparently the most natural way to do so is to replace $\mathscr{T}$ by a suitable CA rule $T$ with very large range. This illustrates an illuminating interplay between the discrete and continuous settings.

\section{FinAl REMARKS ON THE CONNECTION WITH EXCITABLE MEDIA}

Let us conclude by outlining some connections with models for twodimensional excitable media. As mentioned in the introduction, our computer experiments with Greenberg-Hastings and cyclic cellular CA rules, reported in [6], were the original motivation for the present work. The color graphics of that article show examples of periodic wave generation by spiral cores known as stable periodic objects. In many cases the wave fronts evolve as if they were governed by a monotone rule $T$, and hence are close to a suitable $\mathscr{T}$ once the range is sufficiently large. In such instances a corresponding shape $L=K_{1 / w}^{*}$ gives a good approximation to the geometry of the wave fronts.

To convey the spirit of these remarks more precisely, we introduce a Euclidean deterministic forest fire (DFF) on $\mathbb{R}^{2}$ as follows. At each time $n$ the state of the system consists of two disjoint Borel subsets of $\mathbb{R}^{2}: F_{n}$ (points "on fire"), and $E_{n}$ ("burnt" points). The dynamics are

$$
F_{n+1}=\left\{x \in \mathbb{R}^{2} \backslash\left(F_{n} \cup E_{n}\right):\left|\left(F_{n} \cap(x+\mathscr{N})\right)\right| \geq \theta\right\}, \quad E_{n+1}=F_{n} \cup E_{n} .
$$

Here $\theta$ and $\mathscr{N}$ are the same parameters as for $\mathscr{T}$. In words, a point which is neither on fire nor burnt is ignited next time if the volume of overlap between its neighborhood and the currently burning region exceeds $\theta$, and any point remains ignited for a single time unit before it becomes burnt. Theorem 1 has the following consequence for Euclidean DFF dynamics with small threshold.

Corollary 1. Let $\mathscr{N}$ be completely symmetric and convex. Assume that $E_{0}=\varnothing$ and $F_{0}$ is a completely symmetric and convex set that contains $B(R)$ for a suitably large $R($ e.g., $8 \mathscr{N} \subset B(R))$. There exists a $\theta_{0}=\theta_{0}(\mathscr{N})>0$ such that if $\theta<\theta_{0}$, then $n^{-1} E_{n} \stackrel{H}{\rightarrow} L$ as $n \rightarrow \infty$, where $L$ is the limiting shape for $\mathscr{T}=\mathscr{T}_{\boldsymbol{\theta}}, \mathfrak{N}$.

Proof. We will show that $F_{n} \cup E_{n}=\mathscr{T}^{n} F_{0}$ for all $n \geq 0$; once this identity is established the corollary is an immediate consequence of Theorem 1. Observe first that, by Proposition A1(b), $\mathscr{T}^{n} F_{0}$ is completely symmetric and convex for every $n$ provided that $\widehat{\mathscr{T}} A \supset A$. For the sake of brevity, let us write $\varphi$ in place of $u_{\varphi}=\cos \varphi e_{1}+\sin \varphi e_{2}(\varphi \in[0,2 \pi))$. It suffices to verify the following claim for all sufficiently small $\theta>0$ :

If $A$ is a large completely symmetric convex set, and

$$
\lambda_{\varphi}=\sup \{\lambda>0: A \cap(\lambda \varphi+\mathscr{N}) \neq \varnothing\},
$$

then $\mu_{\varphi}=\left|\mathscr{T} A \cap\left(\lambda_{\varphi} \varphi+\mathscr{N}\right)\right| \geq \theta \quad(\varphi \in \mathscr{S})$. Let $a=2 \alpha_{\mathscr{N}}(\pi / 8) \sin (\pi / 8)$. Introduce the quarter-spaces

$$
Q_{\varphi}^{-}=\bigcap\left\{H_{\psi}^{-}: \cos (\varphi-\psi)=1 / \sqrt{2}\right\}, \quad \varphi \in \mathscr{S} .
$$


By the assumed properties of $A$, it suffices to check the claim in two cases. The first is when $A=Q_{\pi / 4}^{-}$and $\varphi \in\left[0, \frac{\pi}{2}\right]$; the second is when $A=Q_{0}^{-}$and $\varphi \in\left[-\frac{\pi}{4}, \frac{\pi}{4}\right]$. To handle the first case, let

$$
\delta=\min \left\{\frac{1}{\sqrt{2}} \alpha_{\mathscr{T} Q_{\pi / 4}^{-}}\left(\frac{\pi}{4}\right), \frac{1}{\sqrt{2}} a\right\} .
$$

Clearly $\delta$ increases with $\theta$ and $\delta>0$ if $\theta$ is small enough. But $\mu_{\varphi} \geq \delta^{2}$ for every $\varphi \in\left[0, \frac{\pi}{2}\right]$. The second case can be dealt with similarly.

We remark that if $\mathscr{N}=B_{\infty}(1)$, then the idea of this last argument yields a $\theta_{0}$ slightly larger than $\frac{1}{4}$. We believe that the result should hold with $\theta_{0}=\frac{1}{2}$, but not for larger $\theta_{0}$. In order to achieve that improvement one would need to substitute half-spaces for the $Q_{\varphi}^{-}$. At larger thresholds than $\frac{1}{2}$ the Euclidean DFF dynamics cease to agree with $\mathscr{T}^{n}$, and entirely new methods are needed for their study.

Similar techniques apply to analogously defined DFF CA rules. Assume for simplicity that $N_{k}=k \mathscr{N} \cap \mathbb{Z}^{2}$, where $\mathscr{N}$ is as in Corollary 1. There exists a constant $c=c(\mathscr{N})$ such that if $\theta_{k}<k^{2} \theta_{0}-c k$, then the following is true. If $F_{0}=B_{\infty}(R) \cap \mathbb{Z}^{2}$ for a sufficiently large $R$, and $E_{0}=\varnothing$, then $n^{-1} E_{n} \stackrel{H}{\rightarrow} L$ as $n \rightarrow \infty$, where $L$ is the limiting shape for $T=T_{\theta, N}$. For example, if $\mathscr{N}=B_{\infty}(1)$, then $\theta_{k} \leq \frac{1}{4} k^{2}-\frac{1}{2} k$ suffices.

Let us now formally define the Greenberg-Hastings model $\xi_{t}$ (GHM) and cyclic cellular automaton $\zeta_{t}$ (CCA), models for excitable media that were mentioned in the Introduction. These are the iterated transformations on $\{0,1 \ldots, \kappa-1\}^{\mathbb{Z}^{2}}, \kappa \geq 3$, that were studied in [6]. Starting from $\xi_{0}$ and $\zeta_{0}$, respectively, the GHM and CCA are defined recursively by

$(\mathrm{GH})$

$$
\begin{aligned}
\xi_{t+1}(x) & =\left(\xi_{t}(x)+1\right) \bmod \kappa & & \text { if } \xi_{t}(x) \geq 1 \\
& =\xi_{t}(x) & & \text { or } \quad \#\left\{y \in x+N: \xi_{t}(y)=1\right\} \geq \theta,
\end{aligned}
$$

$$
\begin{aligned}
\zeta_{t+1}(x) & =\left(\zeta_{t}(x)+1\right) \bmod \kappa & & \text { if } \#\left\{y \in x+N: \zeta_{t}(y)=\left(\zeta_{t}(x)+1\right) \bmod \kappa\right\} \geq \theta \\
& =\zeta_{t}(x) & & \text { otherwise. }
\end{aligned}
$$

The following result for GHM or CCA dynamics started from a lattice ball of 1 's on a background of 0's is essentially a restatement of the discrete version of Lemma 1.

Corollary 2. Assume that $N_{k}$ is as above and $\theta_{k} \leq k^{2} \theta_{0}-c k$. Let the initial state have all 1 's on $B_{\infty}(R)$ and 0 's elsewhere, where $R$ is large. For either GHM or CCA, let $R_{t}=\{x: 1$ at $x$ at time $t\}, S_{t}=\{x: 1$ at $x$ at some time $s \leq t\}$. Then $t^{-1} S_{t} \rightarrow L$ as $t \rightarrow \infty$, and if $\kappa$ is sufficiently large then $t^{-1} R_{t} \rightarrow \alpha_{L}(\mathscr{S})$ (the boundary of $L$ ). Here $L$ is the limit shape of the $\left(\theta_{k}, N_{k}\right)$ threshold growth $C A$.

This last result describes "ring dynamics." In the terminology of [6], for $\mathcal{N}=B_{\infty}(1)$, it states that ball $(k) \geq \frac{1}{4} k^{2}-\frac{1}{2} k$. However it also accurately describes the shapes of wave fronts that one sees in excitable cellular automata started from random initial states provided $\theta$ is sufficiently small. The reason 
is better understood for GHM: stable periodic objects form rapidly, and they invariably produce nested rings provided $\kappa$ is suitably large. These rings must then propagate with the asymptotic shape described in Corollary 2 . Spiral formation in CCA rules is less well understood. Suffice it to say that emergent wave fronts with limiting shape $L$ are observed experimentally in both the locally periodic and turbulent regions of the phase diagram (cf. [6]).

In conclusion, we would like to mention one natural extension of our threshold growth dynamics that may find application in more detailed models for excitable media. Namely, more general functionals might replace the neighbor set $\mathscr{N}$ in the sense that $\mathscr{T} A=\widehat{\mathscr{T}} A \cup A$, where

$$
\widehat{\mathscr{T}} A=\left\{x \in \mathbb{R}^{d}: \int_{A} f(x+y) d y \geq \theta\right\},
$$

i.e., $f=1_{\mathcal{N}}$ is replaced by any $f: \mathbb{R}^{d} \rightarrow[0, \infty)$ such that $\int f<\infty$. After extending the definition of the width function $w$ in a straightforward manner, many techniques and results from this paper apply.

\section{APPENDIX: PreliminARIES AND TEChNICAL DETAILS}

Let us denote the $l^{p}$-ball of radius $r$ centered at $x$ by

$$
B_{p}(x, r)=\left\{y \in \mathbb{R}^{d}:\|y-x\|_{p} \leq r\right\},
$$

$1 \leq p \leq \infty$. We will abbreviate $B_{p}(r)=B_{p}(\mathbf{0}, r)$, and usually omit the subscript when $p=2$. To each vector $u$ in the unit sphere $\mathscr{S}$ there corresponds a half space $H_{u}^{-}$defined by (1.10). The standard basis vectors are written as $e_{i} \quad(1 \leq i \leq d)$. The Borel $\sigma$-algebra on $\mathbb{R}^{d}$ is denoted by $\mathscr{B}\left(\mathbb{R}^{d}\right)$, and we write $|A|$ for the Lebesgue measure of a set $A \in \mathscr{B}\left(\mathbb{R}^{d}\right)$. Also, set \# $A=$ the cardinality of set $A$.

A set $K \subset \mathbb{R}^{d}$ is called star-shaped (with respect to 0 ) if $\{\lambda x: 0 \leq \lambda<1\} \in K$ whenever $x \in K$. For any $f: \mathscr{S} \rightarrow[0, \infty]$, the shape $K_{f}$ is given by $(1.14)$ is clearly star-shaped. On the other hand, if $K \subset \mathbb{R}^{d}$ is any star-shaped set, then its boundary can be represented by a function $\alpha_{K}: \mathscr{S} \rightarrow[0, \infty]$ defined as

$$
\alpha_{K}(u)=\sup \{\lambda \geq 0: \lambda u \in K\} .
$$

Let us now introduce formally the Euclidean and lattice transformations that we will study. Fix neighbor sets $\mathscr{N} \in \mathscr{B}\left(\mathbb{R}^{d}\right)$ with finite volume, and finite $N \subset \mathbb{Z}^{d}$. Also fix a real threshold $\theta>0$. Define $\widehat{\mathscr{T}}: \mathscr{B}\left(\mathbb{R}^{d}\right) \rightarrow 2^{\mathbb{R}^{d}}$, and $\widehat{\mathscr{T}}: 2^{\mathbb{Z}^{d}} \rightarrow 2^{\mathbb{Z}^{d}}$, by

$$
\begin{aligned}
\widehat{\mathscr{T}} A & =\left\{x \in \mathbb{R}^{d}:|(A \cap(x+\mathcal{N}))| \geq \theta\right\}, \\
\widehat{T} B & =\left\{x \in \mathbb{Z}^{d}: \#(B \cap(x+N)) \geq \theta\right\},
\end{aligned}
$$

where $A \in \mathscr{B}\left(\mathbb{R}^{d}\right)$ and $B \subset \mathbb{Z}^{d}$ are arbitrary. In keeping with (1.6)-(1.7), write

$$
\mathscr{T} A=\widehat{\mathscr{T}} A \cup A \text { and } T B=\widehat{T} B \cup B .
$$

Our first proposition identifies some basic properties of these mappings. The definition of obesity appears in $\S 5$ (cf. (5.1)). 
Proposition A1. (a) For any $A \in \mathscr{B}\left(\mathbb{R}^{d}\right), x \mapsto|A \cap(x+\mathscr{N})|$ is a continuous map from $\mathbb{R}^{d}$ to $\mathbb{R}$. Hence $\widehat{\mathscr{T}}$ and $\mathscr{T}$ map $\mathscr{B}\left(\mathbb{R}^{d}\right)$ into $\mathscr{B}\left(\mathbb{R}^{d}\right)$.

(b) Assume that $\mathscr{N}$ is convex. If $A \subset \mathbb{R}^{d}$ is convex, then $\widehat{\mathscr{T}} A$ is convex.

(c) Assume that $N$ is obese. If $A \subset \mathbb{Z}^{d}$ is obese, then so are $\widehat{T} A$ and $T A$.

Proof. For $\varepsilon>0$, define $\mathscr{N}^{\varepsilon}=\mathscr{N}+B(\varepsilon)$, and $\mathscr{N}^{-\varepsilon}=\left(\left(\mathscr{N}^{c}\right)^{\varepsilon}\right)^{c}$. Then $\mathscr{N}^{-\|x\|} \subset x+\mathscr{N} \subset \mathscr{N}^{\|x\|}$. Thus the map in (a) is continuous at $x=0$; a translate of $\mathscr{N}$ handles any $x$.

To prove (b), fix $\lambda \in(0,1)$ and two vectors $x, y \in \mathbb{R}^{d}$, and define the functions

$$
f=1_{(x+\mathscr{N}) \cap A}, \quad g=1_{(y+\mathscr{N}) \cap A}, \quad \text { and } \quad h=1_{(\lambda x+(1-\lambda) y+\mathscr{N}) \cap A} .
$$

By convexity of $\mathscr{N}$ and $A, h(\lambda r+(1-\lambda) s) \geq f(r)^{\lambda} g(s)^{1-\lambda}$ for each $r, s \in \mathbb{R}^{d}$. Hence Lemma 1.2 of Pisier [10] yields

$$
\int h \geq\left(\int f\right)^{\lambda}\left(\int g\right)^{1-\lambda}
$$

If both integrals on the right side exceed $\theta$, then the integral on the left does as well, so $\widehat{\mathscr{T}} A$ is convex.

Under the hypotheses of (c), complete symmetry of $\widehat{T} A$ and $T A$ follow from complete symmetry of counting measure on $\mathbb{Z}^{d}$. Cone condition (5.1(i)) for obesity of $\widehat{T} A$ boils down to

$$
\#\left(\left((x+N) \backslash\left(x-e_{1}+N\right)\right) \cap A\right) \leq \#\left(\left(\left(x-e_{1}+N\right) \backslash(x+N)\right) \cap A\right)
$$

whenever $x_{1}>0$. Let $B$ and $C$ denote the sets being counted on the left and right sides of (A.2), respectively. We claim that $f(y)=y-2\left(y_{1}-x_{1}\right) e_{1}-e_{1}$ is an injection from $B$ to $C$. Obesity implies that each slice $\left\{y \in N: y_{2}, \ldots, y_{d}\right.$ are fixed $\}$ is an interval of integers symmetric about 0 . Thus distinct sites in $B$ must differ in some coordinate other than the first, so $f$ is one-one. Now fix $y \in B$. First note that $y$ must satisfy $y_{1} \geq x_{1}>0$, since obesity of $N$ and $y_{1}<x_{1}$ yield the string of implications: $y \in x+N \Rightarrow x-y \in N \Rightarrow x-y-e_{1} \in$ $N \Rightarrow y \in x-e_{1}+N$. Thus $y \in x+N \Rightarrow y-x-2\left(y_{1}-x_{1}\right) e_{1} \in N \Rightarrow f(y) \in$ $x-e_{1}+N$. Also, $y \notin x-e_{1}+N \Rightarrow f(y) \notin x+N$. Finally, obesity of $A$ implies that $y-c e_{1} \in A$ for $0 \leq c \leq 2 y_{1}$, and $2\left(y_{1}-x_{1}\right)-1 \leq 2 y_{1}$ since $x_{1} \geq 1$. Together these conclusions show that $f(y) \in C$. (A.2) follows. A similar argument verifies (5.1(ii)) for $\widehat{T} A$. Since $A$ and $\widehat{T} A$ are obese, so is $T A$. (Unions of obese sets are obese.)

Repeated application of any one of the four transformations introduced above gives rise to a dynamical system; we use a superscript $n$ to denote the $n$th iterate. Part (a) of Proposition Al ensures that iteration is well defined in the Euclidean setting. In this paper we focus on $\mathscr{T}^{n} ; \S 5$ contains results for $T^{n}$ and a connection between the lattice and Euclidean rules. $\widehat{\mathscr{T}}$ and $\widehat{T}$ are interesting in their own right, but we use them only as auxiliary dynamics in $\S \S 4$ and 6 . Let us note in passing that $\mathscr{T}$ need not preserve convexity when $\mathscr{N}$ is convex; there are easy counterexamples.

As explained in the Introduction, the polar transform (1.13) on sets $K \subset \mathbb{R}^{d}$ plays a key role in our results. The next order of business is to collect needed 
facts about $K^{*}$; most are well known to convex analysts. First, given $K \subset \mathbb{R}^{d}$, define $S_{K}: \mathscr{S} \rightarrow[0, \infty)$ by $S_{K}(u)=\sup \{\langle x, u\rangle: x \in K\}$. Then (1.13) and (A.1) easily imply that

$$
\alpha_{K^{*}}=1 / S_{K}
$$

$\left(\frac{1}{0}=\infty, \frac{1}{\infty}=0\right)$. Assume temporarily that $K$ is compact and convex, with $\mathscr{C}^{1}$ boundary, and $\mathbf{0} \in K$. Let $\nu_{K}: \mathscr{S} \rightarrow \mathscr{S}$ denote the (outward) normal bundle of $K$. Then $\nu_{K}$ is surjective, and $S_{K}\left(\nu_{K}(u)\right)=\alpha_{K}(u)\left\langle u, \nu_{K}(u)\right\rangle$ for each $u \in \mathscr{S}$. Hence

$$
\alpha_{K^{*}}\left(\nu_{K}(u)\right)=\frac{1}{\alpha_{K}(u)\left\langle u, \nu_{K}(u)\right\rangle} \quad \text { for each } u \in \mathscr{S} \text {. }
$$

Comparing (1.12) and (A.5), our heuristic evaluation of the limiting velocities $\alpha_{L}(u)$ should solve

$$
w\left(\nu_{L}(u)\right)=1 / \alpha_{L^{*}}\left(\nu_{L}(u)\right),
$$

or equivalently, $\alpha_{L^{*}}=1 / w$. That is to say, $L^{*}=K_{1 / w}$. As noted below, $K^{* *}=K$ for closed convex sets $K$. Hence, taking the polar of both sides, we get (1.15).

Since our goal is to establish the formula (1.15) even when $L$ is not smooth and $1 / w$ is not convex, we need a less restrictive analog of (A.5). This and other fundamental properties of the general polar transform are collected in the next proposition. $\operatorname{cl}(\cdot)$, in $(\cdot)$, and $\operatorname{co}(\cdot)$ denote the closure, interior, and convex hull of a set, respectively. We abbreviate $\overline{\mathrm{co}}(\cdot)=\operatorname{cl}(\operatorname{col}(\cdot))$.

\section{Proposition A2.}

(a) If $K$ is any subset of $\mathbb{R}^{d}$, then

(i) $K^{*}$ is a convex set that contains $\mathbf{0}$, and $K^{*}=\overline{\operatorname{co}}(K)^{*}$;

(ii) $K^{*}$ contains a neighborhood of $\mathbf{0}$ iff $K$ is bounded;

(iii) $K^{*}$ is bounded iff $\overline{\mathrm{co}}(K)$ contains a neighborhood of $\mathbf{0}$.

(b) Assume that $K$ is star-shaped and unbounded, and that $\alpha_{K}$ is continuous on $\left\{\alpha_{K}<\infty\right\}$. Then $K^{*}$ has nonempty interior iff there is a $u \in \mathscr{S}$ such that $\alpha_{K}(\cdot)<\infty$ on $H_{u}^{-} \cap \mathscr{S}$. If such a $u$ exists,

$$
\operatorname{cl}\left(\left\{u \in \mathscr{S}: \alpha_{K^{*}}(u)>0\right\}\right)=\bigcap\left\{H_{u}^{-}: \alpha_{K}(u)=\infty\right\} \cap \mathscr{S} .
$$

(c) Assume that $K$ is star-shaped and $\alpha_{K}$ is $\mathscr{C}^{1}$. Then (A.4) holds for each $u \in \mathscr{S}$ such that $\alpha_{K}(u)=\alpha_{\operatorname{co}(K)}$, and $\left\{\nu_{K}(u): \alpha_{K}(u)=\alpha_{\operatorname{co}(K)}(u)\right\}=\mathscr{S}$.

(d) Let $K$ be closed and star-shaped. Then the existence of $\nu_{K^{*}}(u)$ for some fixed $u \in \mathscr{S}$ implies that $\alpha_{K}\left(\nu_{K^{*}}(u)\right) \cdot \nu_{K^{*}}(u)$ is an extreme point of $\operatorname{co}(K)$.

(e) If $K$ is convex and contains $\mathbf{0}$, then $D=\left\{u: \nu_{K}(u)\right.$ exists $\}$ is dense in $\mathscr{S}$ and (A.4) holds for $u \in D$.

(f)

(i) If $K$ is closed, convex, and contains $\mathbf{0}$, then $K^{* *}=K$;

(ii) Let $K$ be a bounded star-shaped neighborhood of $\mathbf{0}$. Then $K^{*}$ is strictly convex iff $\alpha_{K}$ is $\mathscr{C}^{1}$, and $K$ is strictly convex iff $\alpha_{K^{*}}$ is $\mathscr{C}^{1}$.

(iii) Assume that $K$ is strictly convex, unbounded, and $\left.\alpha_{K}\right|_{\mathscr{S} \cap H_{u}^{-}}<\infty$ for some $u \in \mathscr{S}$. Then the boundary of $K^{*}$ is $\mathscr{C}^{1}$ away from the origin. Equivalently, there is an $a>0$ such that $a u \in \operatorname{in}\left(K^{*}\right)$ and $\alpha_{K^{*}-a u}$ is $\mathscr{C}^{1}$ on $\mathscr{S} \backslash\{u\}$. 
(g) Let $K_{m} \subset \mathbb{R}^{d} ; m \geq 1$.

(i) If $K_{m} \uparrow K$, and $K^{*}$ is bounded, then $K_{m}^{*} \downarrow K^{*}$ and $K_{m}^{*} \stackrel{H}{\rightarrow} K^{*}$;

(ii) If $K_{m}$ are closed and convex, $K_{m} \downarrow K$, and $K^{*}$ is bounded, then $K_{m}^{*} \stackrel{H}{\rightarrow}$ $K^{*}$.

(iii) Assume that $f, f_{k}: \mathscr{S} \rightarrow[0, \infty)$ are continuous, and $f_{k} \uparrow f$ as $k \rightarrow$ $\infty$. Assume also that $f>0$ on $H_{-e_{1}}^{-} \cap \mathscr{S}$. Then $\overline{\mathrm{co}}\left(K_{1 / f_{k}}\right) \downarrow \overline{\mathrm{co}}\left(K_{1 / f}\right)$ as $k \rightarrow \infty$.

Note. In part (g) and henceforth, $K_{m} \uparrow K$ for subsets of $\mathbb{R}^{d}$ means that $K_{m} \subset$ $K_{m+1}$ for each $m$ and that $K=\bigcup_{m} K_{m}$. Similarly for $K_{m} \downarrow K$. This does not imply convergence in the Hausdorff metric.

Proof. See Istratescu [4] for (a) part (i), (c), (d) and (f). Roberts and Varberg prove (e) in [11]. Parts (a)(ii)-(iii) and (b) follow easily from (A.3), the fact that

$$
S_{K}(v)=\infty \text { whenever } \alpha_{K}(u)=\infty \text { and }\langle u, v\rangle>0,
$$

and the assumed continuity of $\alpha$.

The assertions of part (g) are not quite as standard, so we will outline their proofs. For (i), first note that if $K_{m} \uparrow K$, then $K_{m}^{*} \downarrow K^{*}$. Suppose the $K_{m}^{*}$ do not converge to $K^{*}$ in the Hausdorff metric. By $(\mathrm{a})(\mathrm{i}), K_{m}^{*}$ is convex for each $m$. Then since $K^{*}$ is bounded, we can find $\varepsilon>0, R<\infty$ so that $z_{m} \in\left(K_{m}^{*} \backslash\left(K^{*}\right)^{\varepsilon}\right) \cap B(\mathbf{0}, R)$ for all $m$. Let $z$ be a subsequential limit of the $z_{m}$. For each $m, z \in K_{m}^{*} \cap B(\mathbf{0}, R)$ (closed), and hence $z \in K^{*}$. But each $z_{m}$ is separated from $K^{*}$ by distance at least $\varepsilon$, a contradiction. This proves part (i). We omit the proof of (ii), which is similar once one applies (f)(i) to see that $\left(\bigcup K_{m}^{*}\right)^{*}=K$, and hence $\operatorname{cl}\left(\bigcup K_{m}^{*}\right)=K^{*}$.

Finally, the more intricate argument for (g)(iii) goes as follows. Note first that the $f_{k}$ converge uniformly to $f$ because the $f_{k}$ and $f$ are continuous and the limit is monotone. Hence, for every $\varepsilon>0$, there exists a $\delta>0$ and a $k_{0}$ so that for $k \geq k_{0}$,

$$
\left\{f_{k} \leq \delta\right\} \subset\{f=0\}^{\varepsilon} .
$$

After inversion this becomes

$$
K_{1 / f_{k}} \cap B(1 / \delta)^{c} \subset[0, \infty)\{f=0\}^{\varepsilon} .
$$

By uniform convergence of $\min \left\{1 / f_{k}, 1 / \delta\right\}$, we can enlarge $k_{0}$ if necessary so that for $k \geq k_{0}$,

$$
K_{1 / f_{k}} \cap B(1 / \delta) \subset K_{1 / f}^{\varepsilon} .
$$

Now (A.7) and (A.8) together imply that

$$
K_{1 / f_{k}} \subset\left(\overline{\mathrm{co}}\left(K_{1 / f}\right)+[0, \infty)\{f=0\}^{\varepsilon}\right)^{\varepsilon} .
$$

In view of (A.9) we need to establish the following

Claim. Let $K$ be any closed convex set including 0 such that $\alpha_{K}<\infty$ on $H_{-e_{1}}^{-} \cap \mathscr{S}$. Then for sufficiently small $\varepsilon>0$,

$$
K_{\varepsilon}=K+[0, \infty)\left\{\alpha_{K}=\infty\right\}^{\varepsilon}
$$

is a closed convex set, and $K_{\varepsilon} \downarrow K$ as $\varepsilon \downarrow 0$. 
Assuming the validity of the claim for a moment, let us see how it completes the proof. Set $K=\overline{\overline{c o}}\left(K_{1 / f}\right)$. Clearly, by monotonicity, $K \subset \overline{\mathrm{co}}\left(K_{1 / f_{k}}\right)$ for all $k$. By (A.9) on the other hand, $K_{1 / f_{k}} \subset K_{\varepsilon}^{\varepsilon}$, which is closed and convex since $K_{\varepsilon}$ is closed and convex for small enough $\varepsilon$. Hence $\overline{\operatorname{co}}\left(K_{1 / f_{k}}\right) \subset K_{\varepsilon}^{\varepsilon}$. Finally, $K_{\varepsilon}^{\varepsilon} \downarrow K$ as $\varepsilon \rightarrow 0$, again because the $K_{\varepsilon}$ are closed. Thus $\overline{\operatorname{co}}\left(K_{1 / f_{k}}\right) \downarrow K$, as was to be proved.

Now let us verify the claim. First, choose $m$ large enough that $K \cap B(m)^{c} \subset$ $[0, \infty)\left\{\alpha_{K}=0\right\}^{\varepsilon}$. Then $K_{\varepsilon}=(K \cap B(0, m))+[0, \infty)\left\{\alpha_{K}=0\right\}^{\varepsilon}$. As the sum of a compact set and a closed set, $K_{\varepsilon}$ is evidently closed. So it remains to check convergence. Fix $z \notin K$; assume without loss of generality that $z_{1}>0$ since otherwise $z \notin K^{\varepsilon}$ as soon as $\left\{\alpha_{K}=\infty\right\}^{\varepsilon} \subset\left(H_{-e_{1}}^{-}\right)^{c}$. Let $A=\left\{x: x_{1}=z_{1}\right\} \cap K$. For every $y \in K$ with $y_{1} \leq z_{1}$ and every $u$ such that $\alpha_{K}(u)=\infty$, the ray $y+[0, \infty) u$ intersects $\left\{x_{1}=z_{1}\right\}$ inside $A$. Let us assume that $y+t u \in A$ is such a point of intersection. Then $t=\left(z_{1}-y_{1}\right) /\left\langle u, e_{1}\right\rangle$. Set

$$
b(z)=z_{1}+\min \left\{\left|y_{1}\right|: y \in K \text { and } y_{1} \leq 0\right\}, c_{\varepsilon}=\inf \left\{\left\langle u, e_{1}\right\rangle: u \in\left\{\alpha_{K}=\infty\right\}^{\varepsilon}\right\} .
$$

Clearly $c_{\varepsilon}>c_{0} / 2>0$ if $\varepsilon>0$ is sufficiently small. Thus, if $v \in\left\{\alpha_{K}=\infty\right\}^{\varepsilon}$, $y \in K$ with $y_{1} \leq z_{1}$, and $y+t^{\prime} v \in\left\{x_{1}=z_{1}\right\}$, then $y+t^{\prime} v$ is included in the $\left(2 b(z) / c_{0}^{2} \cdot \varepsilon\right)$-fattening of $A$. Hence, for small enough $\varepsilon, z$ does not belong to $K_{\varepsilon} \cap\left\{x_{1}=z_{1}\right\}$.

Recall that the width function $w$, given by (1.11), comprises the half-plane velocities of Euclidean threshold growth dynamics. Our next task is to derive some basic properties of $w$. When combined with formula (1.15) and Proposition A2, these results will enable us to deduce conclusions about the limiting shape $L$ from regularity properties of the neighbor set $\mathscr{N}$.

Proposition A3. The function $w$ is always continuous. In case $\mathscr{N} \subset \mathbb{R}^{d}$ is a bounded connected open set with continuous boundary $\partial \mathscr{N}$, assume additionally that for each $u \in \mathscr{S}$ and all real $\lambda$,

$$
\sigma(\{x \in \partial \mathscr{N}:\langle x, u\rangle=\lambda\})=0,
$$

where $\sigma$ denotes volume in $d-1$ dimensions. Then $w$ is $\mathscr{C}^{1}$ on the set $\{w>0\}$.

Proof. Define $V: \mathscr{S} \times[0, \infty) \rightarrow[0, \infty)$ by $V(u, \lambda)=\left|\left(H_{u}^{-}-\lambda u\right) \cap \mathscr{N}\right|$. Then $w$ is given implicitly by $V(u, w(u))=\theta$. The proof of continuity is straightforward. To demonstrate differentiability, set $A=A(u, \lambda)=\{x \in$ $\mathcal{N}:\langle x+\lambda u, u\rangle=0\}$. Using (A.10) it is not hard to show that

$$
\frac{\partial V}{\partial \lambda}=\sigma(A) \text { and } \frac{\partial V}{\partial u}(y)=\int_{A}\langle y, x\rangle \sigma(d x),
$$

where $y$ is any vector in the tangent plane at $u \in \mathscr{S}$. Both partial derivatives are clearly continuous since $A$ changes continuously. Moreover, if $w(u)>0$, then $\sigma(A(u, \lambda))>0$ as soon as $V(u, \lambda)=\theta$. An applicatiom of the Implicit Function Theorem finishes the proof. In fact,

$$
\frac{\partial w}{\partial u}(y)=\frac{-\int_{A(u, w(u))}\langle y, x\rangle \sigma(d x)}{\sigma(A(u, w(u))} .
$$

(Similar reasoning shows that for each $k \geq 0, w$ is $\mathscr{C}^{k+1}$ if the boundary of $\mathscr{N}$ is $\mathscr{C}^{k}$.) 
In two dimensions, $K_{1 / w}$ inherits convexity from $\mathscr{N}$ provided that $\mathscr{N}$ is smooth, strictly convex, and satisfies a symmetry assumption. As far as we can tell the proof below does not extend to $d \geq 3$ unless $\mathscr{N}$ is smooth and $\theta$ is less than some small $\theta_{0}(\mathscr{N})$. Indeed, we believe there are counterexamples to the three-dimensional analog of the following result.

Proposition A4. Assume that $d=2$ and $-\mathcal{N}=\mathscr{N}$.

(a) If $\mathscr{N}$ is convex, then $K_{1 / w}$ is convex for every $\theta$.

(b) If $\mathscr{N}$ is strictly convex, then $K_{1 / w}$ is strictly convex for every $\theta$.

Proof. (a) $K_{\alpha}$ is convex iff for each $u, v \in \mathscr{S}$,

$$
\frac{\alpha(u) \alpha(v)}{\alpha(u)+\alpha(v)}\|u+v\| \leq \alpha\left(\frac{u+v}{\|u+v\|}\right) .
$$

Thus we need to prove that

$$
w(u)+w(v) \geq w\left(\frac{u+v}{\|u+v\|}\right) \cdot\|u+v\|,
$$

or equivalently, with $V$ defined as in the proof of Proposition A3, and setting $r=(u+v) /\|u+v\|$,

$$
V(u, \lambda)=\theta \quad \text { and } \quad V(v, \mu)=\theta \Rightarrow V\left(r, \frac{\lambda+\mu}{\|u+v\|}\right) \leq \theta .
$$

Given $\lambda \neq \mu$, choose $x$ so that $\langle x, u\rangle=\lambda$ and $\langle x, v\rangle=\mu$. Let $l_{0}$ be the line containing 0 and $x$, and for $\varphi \in[0, \pi]$, let $l_{\varphi}$ be the line that makes angle $\varphi$ with respect to $l_{0}$. (Choose the normal to $l_{0}$ in some canonical way to ensure uniqueness of $l_{\varphi}$.) Now define $F:[0, \pi] \rightarrow[0, \infty)$ by

$$
F(\varphi)=\mid \text { the component of }\left(\mathscr{N} \cap l_{\varphi}\right)^{c} \text { that does not include } \mathbf{0} \mid \text {. }
$$

To show (A.11), it suffices to check that $F$ cannot have a local maximum in $(0, \pi)$. Clearly we can assume that $x \in \mathscr{N}$. Also, without loss of generality, suppose that $\mathscr{N}$ is strictly convex with differentiable boundary. (A straightforward approximation then handles the general convex case.) With $b, c, \beta$ and $\gamma$ as indicated in Figure 6 below, we have

$$
\frac{d F}{d \varphi}=\frac{1}{2}\left(b(\varphi)^{2}-c(\varphi)^{2}\right), \quad \frac{d^{2} F}{d \varphi^{2}}=-\left(b(\varphi)^{2} \operatorname{cotan} \beta(\varphi)+c(\varphi)^{2} \operatorname{cotan} \gamma(\varphi)\right) .
$$

By strict convexity and symmetry of $\mathcal{N}, \operatorname{cotan} \beta+\operatorname{cotan} \gamma<0$ for every $\varphi$. Thus $F^{\prime \prime}(\varphi)>0$ whenever $F^{\prime}(\varphi)=0$ and the only critical point is a local minimum. Claim (a) follows. Now assume that $1 / w$ is not strictly convex. By suitably rotating $\mathscr{N}$ we can assume that $w(\varphi)=w(0) \cos \varphi$ in some neighborhood of $\varphi=0$, where $\varphi$ is the polar coordinate. But then the function $F$ above has derivative 0 over an open subinterval of $(0, \pi)$. As we have already seen, this is impossible.

In conclusion, let us mention some implications of Propositions A2-A4 for the limiting shape $L$ of threshold growth dynamics when formula (1.15) applies. Propositions A2(b) and A3 ensure that $L$ is $d$-dimensional provided (1.16) holds, precisely the hypothesis we need in our proof of Theorem 1 . Under this assumption, Proposition A2(a)-(b) implies that $L$ is always a convex set containing $\mathbf{0}$, and either $L$ contains a neighborhood of $\mathbf{0}$ or the closure of 


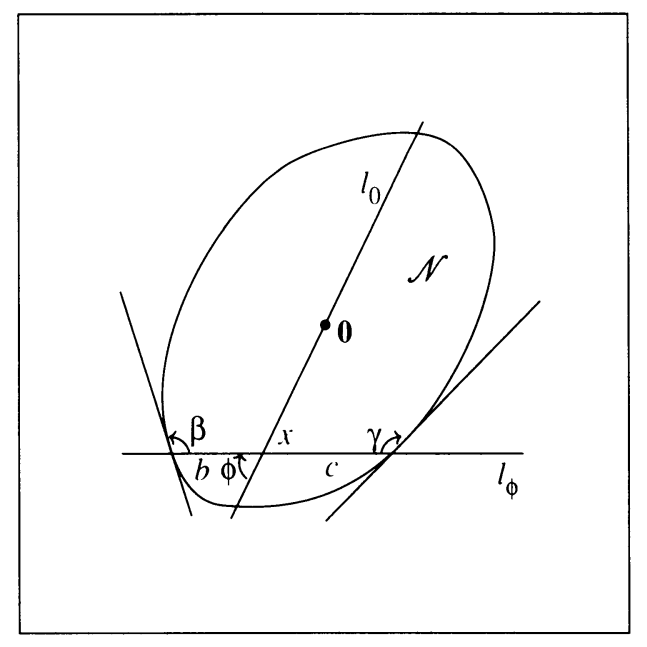

FigURE 6

the support of $\alpha_{L}$ is a "generalized cone" formed by an intersection of halfspaces $H_{u}^{-}$. Propositions A2(c) and A3 then provide a general criterion for the validity of computational formula (A.4). Similarly Proposition A2(d) can be applied to identify directions in which $\alpha_{L}$ is not differentiable.

Two particularly nice results follow from Propositions A2(f), A3, and A4(b). Assume that $\mathscr{N}$ is bounded, connected and open, with continuous boundary, that (A.10) holds, and $w(u)>0$ for all $u$. Then in any $d \geq 2, L$ is strictly convex. If $d=2$, and $\mathscr{N}$ is closed, strictly convex and symmetric $(-\mathcal{N}=\mathscr{N})$, then the boundary of $L$ is $\mathscr{C}^{1}$. Two-dimensional examples such as those in $\S 2$ show that the boundary of $L$ can have flat regions if $w(u)=0$ in certain directions, and the boundary of $L$ can have "corners" if the boundary of $\mathscr{N}$ has flat regions.

\section{REFERENCES}

1. J. D. Biggins, The asymptotic shape of branching random walk, Adv. Appl. Prob. 10 (1978), 62-84.

2. R. Durrett, Lecture notes on particle systems and percolation, Wadsworth \& Brooks/Cole, Pacific Grove, Calif., 1988.

3. M. Eden, A two dimensional growth process, Volume IV of Proc. 4th Berkeley Sympos., Univ. of California Press, Berkeley, 1961.

4. V. I. Istratescu, Strict convexity and complex strict convexity, Marcel Dekker, 1984.

5. R. Fisch and D. Griffeath, EXCITE!: a periodic wave modeling environment. 1991 (1 disk for IBM-compatibles with VGA graphics), Freeware, Write: D. Griffeath, Mathematics Dept., University of Wisconsin, Madison WI 53706, USA.

6. R. Fisch, J. Gravner, and D. Griffeath, Threshold-range scaling of excitable cellular automata, Statistics and Computing 1 (1991) 23-39.

7. M. Gardner, The fantastic combinations of John Conway's new solitaire game "Life," Scientific Amer. 223:4 (1970), 120-123.

8. J. Greenberg, B. Hassard, and S. Hastings, Pattern formation and periodic structures in systems modeled by reaction-diffusion equations, Bull. Amer. Math. Soc. 84 (1978), 12961327. 
9. G. Lawler, M. Bramson, and D. Griffeath, Internal diffusion limited aggregation, Ann. Probab. 20 (1992), 2117-2140.

10. G. Pisier, The volume of convex bodies and Banach space geometry, Cambridge Univ. Press, Cambridge, 1989.

11. A. Roberts and D. Varberg, Convex functions, Academic Press, New York, 1973.

12. T. Toffoli and N. Margolus, Cellular automata machines, MIT Press, Cambridge, Mass., 1987.

13. N. Weiner and A. Rosenbluth, The mathematical formulation of the problem of conduction of impulses in a network of connected excitable elements, specifically in cardiac muscle, Arch. Inst. Cardiol. Mexico 16 (1946), 205-265.

Department of Mathematics, Cornell University, Ithaca, New York 14853

Current address: Department of Mathematics, University of California, Davis, California 95616

E-mail address: gravner@ucdmath.ucdavis .edu

Department of Mathematics, University of Wisconsin, Madison, Wisconsin 53706

E-mail address: griffeat @math.wisc.edu 